\title{
PRODUCT RIGIDITY IN VON NEUMANN AND C*-ALGEBRAS VIA S-MALLEABLE DEFORMATIONS
}

\author{
DANIEL DRIMBE
}

\begin{abstract}
We provide a new large class of countable icc groups $\mathcal{A}$ for which the product rigidity result from CdSS15] holds: if $\Gamma_{1}, \ldots, \Gamma_{n} \in \mathcal{A}$ and $\Lambda$ is any group such that $L\left(\Gamma_{1} \times \cdots \times \Gamma_{n}\right) \cong L(\Lambda)$, then there exists a product decomposition $\Lambda=\Lambda_{1} \times \cdots \times \Lambda_{n}$ such that $L\left(\Lambda_{i}\right)$ is stably isomorphic to $L\left(\Gamma_{i}\right)$, for any $1 \leq i \leq n$. Class $\mathcal{A}$ consists of groups $\Gamma$ for which $L(\Gamma)$ admits an s-malleable deformation in the sense of Sorin Popa and it includes all non-amenable groups $\Gamma$ such that either (a) $\Gamma$ admits an unbounded 1-cocycle into its left regular representation, or (b) $\Gamma$ is an arbitrary wreath product group with amenable base. As a byproduct of these results, we obtain new examples of $\mathrm{W}^{*}$-superrigid groups and new rigidity results in the $\mathrm{C}^{*}$-algebra theory.
\end{abstract}

\section{INTRODUCTION}

Every countable group $\Gamma$ gives rise to the group von Neumann algebra $L(\Gamma)$ by considering the weak operator closure of the complex group algebra $\mathbb{C}[\Gamma]$ acting on the Hilbert space $\ell^{2}(\Gamma)$ by left convolution MvN43. A main theme in operator algebras is the classification of group von Neumann algebras which is centered around the following question: what properties of the group $\Gamma$ are remembered by $L(\Gamma)$ ? This problem is the most interesting when $\Gamma$ is icc (i.e., all non-trivial conjugacy classes of $\Gamma$ are infinite), which corresponds to $L(\Gamma)$ being a $\mathrm{II}_{1}$ factor. In the amenable case, the classification is completed by the work of Connes [Co76] which asserts that any two icc amenable groups give rise to isomorphic von Neumann algebras. Therefore, besides the amenability of the group, no information can be recovered from $L(\Gamma)$ when $\Gamma$ is icc amenable.

In the non-amenable case the situation is radically different and far more complex. An outstanding progress has been achieved since the invention of Popa's deformation/rigidity theory [Po07] and there have been discovered many instances when various algebraic and analytical properties of a group $\Gamma$ can be recovered from $L(\Gamma)$, see the surveys [Va10a, Io12b, Io17. Remarkably, Ioana, Popa and Vaes found in [IPV10] the first class of countable groups $\Gamma$ that are $\mathrm{W}^{*}$-superrigid. Roughly speaking, this means that the group $\Gamma$ is completely remembered by its von Neumann algebra $L(\Gamma)$. Subsequently, several other classes of $\mathrm{W}^{*}$-superrigid groups have been found $\mathrm{BV} 12$, Be14, CI17, CD-AD20.

However, in general, one can only expect to recover certain aspects of a group $\Gamma$ from its von Neumann algebra $L(\Gamma)$. We only highlight the following developments. Ozawa showed in Oz03] that the group von Neumann algebra of a non-amenable bi-exact icc group is prime, in particular implying that $L(\Gamma) \varsubsetneqq L\left(\Gamma_{1} \times \Gamma_{2}\right)$, for all infinite groups $\Gamma_{1}$ and $\Gamma_{2}$. Ozawa and Popa then proved that any tensor product of $\mathrm{II}_{1}$ factors of non-amenable hyperbolic icc groups admits a unique prime decomposition into prime factors [OP03]. As a corollary, their result shows that if $L\left(\Gamma_{1} \times \cdots \times \Gamma_{n}\right) \cong$ $L\left(\Lambda_{1} \times \cdots \times \Lambda_{m}\right)$ for some icc hyperbolic groups $\Gamma_{i}$ 's and infinite groups $\Lambda_{j}$ 's, then $m=n$ and after a permutation of indices we have $L\left(\Gamma_{i}\right)$ is stably isomorphic to $L\left(\Lambda_{i}\right)$, for all $1 \leq i \leq n$. More recently, Chifan, de Santiago and Sinclair strengthened the previous corollary of OP03 by discovering the

The author holds the postdoctoral fellowship fundamental research $12 \mathrm{~T} 5221 \mathrm{~N}$ of the Research Foundation Flanders. 
DANIEL DRIMBE

following product rigidity phenomenon: if the groups $\Gamma_{i}$ 's are icc hyperbolic, then any group $\Lambda$ such that $L\left(\Gamma_{1} \times \cdots \times \Gamma_{n}\right) \cong L(\Lambda)$ admits a decomposition $\Lambda=\Lambda_{1} \times \cdots \times \Lambda_{n}$ satisfying $L\left(\Gamma_{i}\right)$ is stably isomorphic to $L\left(\Lambda_{i}\right)$, for any $1 \leq i \leq n$ [CdSS15. While a large number of other unique prime factorization results have been obtained since [OP03] (see, e.g., the introduction of [DHI16]), the above product rigidity result has been extended to the class of non-amenable bi-exact groups only very recent CD-AD20]. Some of the methods from CD-AD20, including an augmentation technique, will play an important role in our work as well.

The goal of this paper is to provide a new class of countable groups, denoted Class $\mathcal{A}$, for which the above product rigidity holds, see Theorem A. A common feature of these groups is that their von Neumann algebras admit an s-malleable deformation in the sense of Popa [Po01, Po03] (see Definition 3.1) and a key ingredient of the proof of our first main result is the use of Popa's spectral gap principle that was developed in $\mathrm{Po06a}, \mathrm{Po} 06 \mathrm{~b}$. In fact, Theorem $\mathrm{A}$ follows from a more general and conceptual result, see Theorem 5.2 .

Class $\mathcal{A}$. We say that a countable non-amenable icc group $\Gamma$ belongs to Class $\mathcal{A}$ if $\Gamma$ satisfies one of the following conditions:

(1) $\Gamma$ admits an unbounded cocycle for some mixing representation $\pi: \Gamma \rightarrow \mathcal{O}\left(H_{\mathbb{R}}\right)$ such that $\pi$ is weakly contained in the left regular representation of $\Gamma$.

(2) $\Gamma=\Gamma_{1} *_{\Sigma} \Gamma_{2}$ is an amalgamated free product group satisfying $\left[\Gamma_{1}: \Sigma\right] \geq 2$ and $\left[\Gamma_{2}: \Sigma\right] \geq 3$, where $\Sigma<\Gamma$ is an amenable almost malnormal 1 subgroup.

(3) $\Gamma=\Sigma \imath_{G / H} G$ is a generalized wreath product group with $\Sigma$ amenable, $G$ non-amenable and $H<G$ is an amenable almost malnormal subgroup.

For any $i \in \overline{1,3}$, if $\Gamma$ belongs to $\mathcal{A}$ satisfying condition (i), then we say that $\Gamma$ belongs to $\mathcal{A}_{i}$. Note that $\mathcal{A}_{1}$ contains all non-amenable icc groups $\Gamma$ with $\beta_{1}^{(2)}(\Gamma)>0$.

Theorem A. Let $\Gamma=\Gamma_{1} \times \cdots \times \Gamma_{n}$ be a product of $n \geq 1$ countable groups that belong to $\mathcal{A}$ and denote $M=L(\Gamma)$. Let $\Lambda$ be any countable group such that $M^{t}=L(\Lambda)$ for some $t>0$.

Then there exist a product decomposition $\Lambda=\Lambda_{1} \times \cdots \times \Lambda_{n}$, a unitary $u \in \mathcal{U}\left(M^{t}\right)$ and some positive numbers $t_{1}, \ldots, t_{n}$ with $t_{1} \ldots t_{n}=t$ such that $u L\left(\Lambda_{i}\right) u^{*}=L\left(\Gamma_{i}\right)^{t_{i}}$, for any $i \in \overline{1, n}$.

Remark that CdSS15, Theorem A] shows that if the groups $\Gamma_{i}$ 's are non-amenable free groups and $L\left(\Gamma_{1} \times \cdots \times \Gamma_{n}\right) \cong L(\Lambda)$ for some group $\Lambda$, then $\Lambda$ admits a product decomposition into $n$ infinite groups. Theorem $\mathrm{A}$ strengthens this fact by replacing the groups $\Gamma_{i}$ 's with the more general class of arbitrary free product groups.

To put our result into a better perspective, we note that Popa's deformation/rigidity theory led to striking rigidity results for group von Neumann algebras of wreath product groups. To recall this results, fix a non-trivial abelian group $A$. Popa showed in [Po03, Po04] that the group von Neumann algebras $L\left(A<\Gamma_{1}\right)$ are pairwise non-isomorphic for different icc property $(\mathrm{T})$ groups $\Gamma_{1}$. This result was strengthened by Ioana, Popa and Vaes in [IPV10] by showing that for any icc property (T) group $\Gamma_{1}$, the isomorphism $L\left(A \prec \Gamma_{1}\right) \cong L(\Lambda)$ implies that there exists a semi-direct product decomposition $\Lambda=B \rtimes \Lambda_{1}$ such that $\Gamma_{1} \cong \Lambda_{1}$. Several other rigidity results have been obtained for group von Neumann algebras of (generalized) wreath product groups, including primeness, unique prime factorization and relative solidity, see [Io06, Po06a, CI08, IPV10, SW11, BV12, IM19]. Theorem $\mathrm{A}$ provides on the other hand a new general rigidity result for wreath product groups by showing that the von Neumann algebra of a product of wreath product groups with amenable base completely remembers the product structure.

\footnotetext{
${ }^{1}$ A subgroup $H<G$ is called almost malnormal if $g H g^{-1} \cap H$ is finite for any $g \in G \backslash H$.
} 
Remark 1.1. We also emphasize the following new rigidity phenomenon that Theorem $\mathrm{A}$ (more precisely, Theorem 5.2) leads to. First, we consider the class $\mathcal{M}_{0}$ of all non-amenable $\mathrm{II}_{1}$ factors $M$ that admit an s-malleable deformation $\left(\tilde{M},\left(\alpha_{t}\right)_{t \in \mathbb{R}}\right)$ with the properties that $M \subset \tilde{M}$ is mixing, ${ }_{M} L^{2}(\tilde{M}) \ominus L^{2}(M)_{M}$ is weakly contained in the coarse bimodule ${ }_{M} L^{2}(M) \otimes L^{2}(M)_{M}$ and $\alpha_{t}$ does not converge uniformly on $(M)_{1}$ (see also Subsection 3.2). In Theorem 5.2 we classify all tensor product decompositions of any group von Neumann algebra for which the tensor factors belong to $\mathcal{M}_{0}$. In contrast to the product rigidity result from CdSS15, the $\mathrm{II}_{1}$ factors from $\mathcal{M}_{0}$ are not necessarily group von Neumann algebras. For instance, any tracial non-amenable free product $M_{1} * M_{2}$ belongs to $\mathcal{M}_{0}$, see Remark 3.6.

Next, we show that Theorem A can be used together with [PV10] to derive new examples of $\mathrm{W}^{*}$-superrigid product groups. First, recall that a countable group $\Gamma$ is $W^{*}$-superrigid if for any group $\Lambda$ and any *-isomorphism $\theta: L(\Gamma)^{t} \rightarrow L(\Lambda)$ for some $t>0$, we have $t=1$ and there exist a group isomorphism $\delta: \Gamma \rightarrow \Lambda$, a character $\omega: \Gamma \rightarrow \mathbb{T}$ and a unitary $w \in L(\Lambda)$ such that $\theta\left(u_{g}\right)=\omega(g) w v_{\delta(g)} w^{*}$, for any $g \in \Gamma$. Here, we denoted by $\left\{u_{g}\right\}_{g \in \Gamma}$ and $\left\{v_{\lambda}\right\}_{\lambda \in \Lambda}$ the canonical generating unitaries of $L(\Gamma)$ and $L(\Lambda)$, respectively.

The first class of $\mathrm{W}^{*}$-superrigid product groups has recently been found in [CD-AD20] by considering products of $\mathrm{W}^{*}$-superrigid groups from [IPV10 that are bi-exact. As a consequence of Theorem A we can actually drop the bi-exactness assumption and therefore obtain that all products of $\mathrm{W}^{*}$ superrigid groups from [IPV10] are again $\mathrm{W}^{*}$-superrigid. To illustrate our result, we introduce the following class of groups that was considered in [IPV10].

Class $\mathcal{I P \mathcal { V }}$. We say that a countable group $\Gamma$ belongs to Class $\mathcal{I P} \mathcal{V}$ if $\Gamma=(\mathbb{Z} / n \mathbb{Z}) \imath_{I} G$ is a generalized wreath product group that satisfies:

- $n \in\{2,3\}$ and $I=G / H$, where $H<G$ is an infinite amenable almost malnormal subgroup.

- $G$ admits an infinite normal subgroup that either has relative property $(\mathrm{T})$ or its centralizer is non-amenable.

Corollary B. If $\Gamma=\Gamma_{1} \times \cdots \times \Gamma_{n}$ is a product of $W^{*}$-superrigid groups that belong to $\mathcal{A}$ (e.g., $\Gamma_{i} \in \mathcal{I P} \mathcal{V}$ for any $\left.i\right)$, then $\Gamma$ is $W^{*}$-superrigid.

The problem of proving that the $\mathrm{W}^{*}$-superrigid property is closed with respect to direct products is notoriously hard and remains open. In Corollary B (see also Theorem 5.2) we make some progress on this problem, by showing that within the class of generalized wreath product groups with almost malnormal stabilizers, the $\mathrm{W}^{*}$-superrigidity property is preserved by taking direct products.

Finally, we will discuss some applications of Theorem $\mathrm{A}$ to the $\mathrm{C}^{*}$-algebra theory. In contrast to the von Neumann algebra setting, the classification of reduced $C^{*}$-algebras is not governed by an amenable/non-amenable dichotomy in the sense that the reduced group $\mathrm{C}^{*}$-algebra $C_{r}^{*}(\Gamma)$ of an amenable group $\Gamma$ does not provide the same striking lack of rigidity as its von Neumann algebra $L(\Gamma)$. In fact, any torsion free abelian group $\Gamma$ is $C^{*}$-superrigid [Sc74, Theorem 1], which roughly means that $C_{r}^{*}(\Gamma)$ completely remembers the group $\Gamma$. By building upon the result of [Sc74], several other classes of amenable $\mathrm{C}^{*}$-superrigid groups have been found, see the introduction of [ER18]. In the non-amenable case, the only examples of $\mathrm{C}^{*}$-superrigid groups are obtained in CI17,CD-AD20] via Popa's deformation/rigidity theory using their von Neumann algebraic superrigid behavior combined with the unique trace property [BKKO14] of their reduced $\mathrm{C}^{*}$-algebra. In a similar way, the von Neumann rigidity result from Theorem $\mathrm{A}$ can be transferred to a product rigidity in $C^{*}$-algebras.

Corollary C. Let $\Gamma=\Gamma_{1} \times \cdots \times \Gamma_{n}$ be a product of $n \geq 1$ countable non-amenable icc groups such that $\beta_{1}^{(2)}\left(\Gamma_{i}\right)>0$ or $\Gamma_{i} \in \mathcal{A}_{2}$, for any $i \in \overline{1, n}$. Let $\Lambda$ be any countable group satisfying $C_{r}^{*}(\Gamma)=C_{r}^{*}(\Lambda)$. 
DANIEL DRIMBE

Then $\Lambda=\Lambda_{1} \times \cdots \times \Lambda_{n}$ admits a product decomposition into infinite groups such that $L\left(\Lambda_{i}\right)$ is stably isomorphic to $L\left(\Gamma_{i}\right)$, for any $i \in \overline{1, n}$.

We note that the above product rigidity has already been obtained for icc hyperbolic groups [CdSS15] and extended to infinite direct sums of icc hyperbolic property (T) groups [CU18. Finally, it would be interesting to show that in certain cases the conclusion of Corollary [C] can be strengthened to derive that the $\mathrm{C}^{*}$-algebras of the factors $C_{r}^{*}\left(\Gamma_{i}\right)$ and $C_{r}^{*}\left(\Lambda_{i}\right)$ are isomorphic for any $i \in \overline{1, n}$.

Outline of the proof of Theorem $\mathbf{A}$. We outline briefly and informally the proof of our main result, which is Theorem A. Let $\Gamma=\Gamma_{1} \times \cdots \times \Gamma_{n}$ be a product of $n \geq 1$ countable groups that belong to Class $\mathcal{A}$; to better illustrate the proof, we assume that $\beta_{1}^{(2)}\left(\Gamma_{i}\right)>0$, for any $i \in \overline{1, n}$. Denote $M_{i}=L\left(\Gamma_{i}\right)$, for any $i \in \overline{1, n}$ and let $M=L(\Gamma)$.

Our goal is to show that for any group von Neumann algebra decomposition $M^{t}=L(\Lambda)$, where $t>0$ and $\Lambda$ is a countable group, the underlying group is a product group $\Lambda=\Lambda_{1} \times \cdots \times \Lambda_{n}$. To simplify notation, we assume $t=1$. In order to attain this goal, we use a number of techniques from Popa's deformation/rigidity theory. Following [PV09, we define the $*$-homomorphism $\Delta: M \rightarrow M \bar{\otimes} M$ by letting $\Delta\left(v_{\lambda}\right)=v_{\lambda} \otimes v_{\lambda}$, for any $\lambda \in \Lambda$.

Throughout the proof we repeatedly use Popa's spectral gap rigidity principle together with the smalleable deformation constructed by Sinclair [Si10] for all $L\left(\Gamma_{i}\right)$ 's. Their first combined application shows that

$$
\forall i \in \overline{1, n}, \exists t_{i} \in \overline{1, n} \quad \text { such that } \quad \Delta\left(M_{\widehat{i}}\right) \prec M \bar{\otimes} M_{\widehat{t_{i}}} .
$$

Here, $P \prec Q$ denotes that a corner of $P$ embeds into $Q$ inside the ambient algebra, in the sense of Popa [Po03] and $M_{\hat{i}}=\bar{\otimes}_{j \neq i} M_{j}$. Next, by combining Ioana's ultrapower technique [Io1] with some recent techniques in the framework of von Neumann algebras without property Gamma [BMO19, IM19, we are able to upgrade (1.1) to

$$
\Delta\left(M_{I}\right) \prec M \bar{\otimes} M_{I}, \quad \text { for any } I \subset \overline{1, n},
$$

We also derive that for any subset $I \subset \overline{1, n}$ there exists a subgroup $\Sigma_{I}<\Lambda$ with non-amenable centralizer $C_{\Lambda}(\Sigma)$ such that

$$
L\left(\Sigma_{I}\right) \prec M_{I} \quad \text { and } \quad M_{I} \prec L\left(\Sigma_{I}\right) .
$$

Here, we denoted by $M_{I}=\bar{\otimes}_{i \in I} M_{i}$. This is achieved in Section 4 . Next, by using some general recent results on s-malleable deformations dSHHS20 and an augmentation technique developed in [CD-AD20] we are able to use (1.2) and (1.3) to show that there exists a non-zero projection $e \in L\left(\Sigma_{\widehat{n}}\right)^{\prime} \cap M$ such that

$$
L\left(\Sigma_{\widehat{n}}\right) e \prec^{s} M_{\hat{n}} \quad \text { and } \quad M_{\hat{n}} \prec L\left(\Sigma_{\widehat{n}}\right) e .
$$

Here, we denoted by $P \prec^{s} Q$ to indicate that $P p^{\prime} \prec Q$ for any non-zero projection in the relative commutant of $P$. Relation (1.3) shows in particular that

$$
L\left(\Sigma_{n}\right) \prec M_{n} \quad \text { and } \quad M_{n} \prec L\left(\Sigma_{n}\right) .
$$

Finally, by building upon some results from [DHI16], we show that (1.4) and (1.5) imply that there exists a product decomposition $\Lambda=\Lambda_{\widehat{n}} \times \Lambda_{n}$ such that $M_{\widehat{n}}=L\left(\Lambda_{\widehat{n}}\right)$ and $M_{n}=L\left(\Lambda_{n}\right)$, modulo unitary conjugacy and amplifications. The result follows now by induction.

Organization of the paper. Besides the introduction there are four other sections in the paper. In Section 2 we review Popa's intertwining-by-bimodules techniques and some other tools. In Section 3 we recall some general properties of s-malleable deformations and some structural results for $L(\Gamma)$, when $\Gamma \in \mathcal{A}$. Next, in Section 4 we review Ioana's ultrapower technique and present 
an important corollary which, besides being of independent interest, will be used in the proof of Theorem A. Finally, in Section 5 we prove our main results.

Acknowledgments. I am grateful to Adrian Ioana for helpful comments and to the anonymous referees for their many remarks which greatly improved the exposition of the paper.

\section{Preliminaries}

2.1. Terminology. In this paper we consider tracial von Neumann algebras $(M, \tau)$, i.e. von Neumann algebras $M$ equipped with a faithful normal tracial state $\tau: M \rightarrow \mathbb{C}$. This induces a norm on $M$ by the formula $\|x\|_{2}=\tau\left(x^{*} x\right)^{1 / 2}$, for all $x \in M$. We will always assume that $M$ is a separable von Neumann algebra, i.e. the $\|\cdot\|_{2}$-completion of $M$ denoted by $L^{2}(M)$ is separable as a Hilbert space. We denote by $\mathcal{Z}(M)$ the center of $M$ and by $\mathcal{U}(M)$ its unitary group. For two von Neumann subalgebras $P_{1}, P_{2} \subset M$, we denote by $P_{1} \vee P_{2}=W^{*}\left(P_{1} \cup P_{2}\right)$ the von Neumann algebra generated by $P_{1}$ and $P_{2}$.

All inclusions $P \subset M$ of von Neumann algebras are assumed unital. We denote by $E_{P}: M \rightarrow P$ the unique $\tau$-preserving conditional expectation from $M$ onto $P$, by $e_{P}: L^{2}(M) \rightarrow L^{2}(P)$ the orthogonal projection onto $L^{2}(P)$ and by $\left\langle M, e_{P}\right\rangle$ the Jones' basic construction of $P \subset M$. We also denote by $P^{\prime} \cap M=\{x \in M \mid x y=y x$, for all $y \in P\}$ the relative commutant of $P$ in $M$ and by $\mathcal{N}_{M}(P)=\left\{u \in \mathcal{U}(M) \mid u P u^{*}=P\right\}$ the normalizer of $P$ in $M$. We say that $P$ is regular in $M$ if the von Neumann algebra generated by $\mathcal{N}_{M}(P)$ equals $M$. Next, we define the quasi-normalizer $\mathcal{Q N}_{M}(P)$ as the subgroup of all elements $x \in M$ for which there exist $x_{1}, \ldots, x_{n} \in M$ such that $P x \subset \sum_{i} x_{i} P$ and $x P \subset \sum_{i} P x_{i}$ (see [Po99, Definition 4.8]).

The amplification of a $\mathrm{II}_{1}$ factor $(M, \tau)$ by a number $t>0$ is defined to be $M^{t}=p\left(\mathbb{B}\left(\ell^{2}(\mathbb{Z})\right) \bar{\otimes} M\right) p$, for a projection $p \in \mathbb{B}\left(\ell^{2}(\mathbb{Z})\right) \bar{\otimes} M$ satisfying $(\operatorname{Tr} \otimes \tau)(p)=t$. Here $\operatorname{Tr}$ denotes the usual trace on $\mathbb{B}\left(\ell^{2}(\mathbb{Z})\right)$. Since $M$ is a $\mathrm{II}_{1}$ factor, $M^{t}$ is well defined. Note that if $M=P_{1} \bar{\otimes} P_{2}$, for some $\mathrm{II}_{1}$ factors $P_{1}$ and $P_{2}$, then there exists a natural identification $M=P_{1}^{t} \bar{\otimes} P_{2}^{1 / t}$, for every $t>0$.

For a countable group $\Gamma$ and for two subsets $S, T \subset \Gamma$, we denote by $C_{S}(T)=\{g \in S \mid g h=$ $h g$, for all $h \in T\}$ the centralizer of $T$ in $S$. Given a group inclusion $\Sigma<\Gamma$, the quasi-normalizer $\mathrm{QN}_{\Gamma}(\Sigma)$ is the group of all $g \in \Gamma$ for which exists a finite set $F \subset \Gamma$ such that $\Sigma g \subset F \Sigma$ and $g \Sigma \subset \Sigma F$; equivalently, $g \in \mathrm{QN}_{\Gamma}(\Sigma)$ if and only if $\left[\Sigma: g \Sigma g^{-1} \cap \Sigma\right]<\infty$ and $\left[\Sigma: g^{-1} \Sigma g \cap \Sigma\right]<\infty$.

Finally, for a positive integer $n$, we denote by $\overline{1, n}$ the set $\{1, \ldots, n\}$. For any subset $S \subset \overline{1, n}$ we denote its complement by $\widehat{S}=\overline{1, n} \backslash S$. If $S=\{i\}$, we will simply write $\hat{i}$ instead of $\widehat{\{i\}}$. Also, given any product group $G=G_{1} \times \cdots \times G_{n}$ and any tensor product $M_{1} \bar{\otimes} \ldots \bar{\otimes} M_{n}$, we will denote their subproduct supported on $S$ by $G_{S}=\times_{i \in S} G_{i}$ and $M_{S}=\bar{\otimes}_{i \in S} M_{i}$, respectively.

2.2. Intertwining-by-bimodules. We next recall from [Po03, Theorem 2.1 and Corollary 2.3] the powerful intertwining-by-bimodules technique of S. Popa.

Theorem 2.1 ([Po03]). Let $(M, \tau)$ be a tracial von Neumann algebra and $P \subset p M p, Q \subset q M q$ be von Neumann subalgebras. Let $\mathcal{U} \subset \mathcal{U}(P)$ be a subgroup such that $\mathcal{U}^{\prime \prime}=P$.

Then the following are equivalent:

(1) There exist projections $p_{0} \in P, q_{0} \in Q, a *$-homomorphism $\theta: p_{0} P p_{0} \rightarrow q_{0} Q q_{0}$ and a non-zero partial isometry $v \in q_{0} M p_{0}$ such that $\theta(x) v=v x$, for all $x \in p_{0} P p_{0}$.

(2) There is no sequence $\left(u_{n}\right)_{n} \subset \mathcal{U}$ satisfying $\left\|E_{Q}\left(x u_{n} y\right)\right\|_{2} \rightarrow 0$, for all $x, y \in M$.

If one of the equivalent conditions of Theorem 2.1 holds true, we write $P \prec_{M} Q$, and say that $a$ corner of $P$ embeds into $Q$ inside $M$. 
Notation 2.2. Throughout the paper we will use the following notation.

- If $P p^{\prime} \prec_{M} Q$ for any non-zero projection $p^{\prime} \in P^{\prime} \cap p M p$, then we write $P \prec_{M}^{s} Q$.

- If $P \prec_{M} Q q^{\prime}$ for any non-zero projection $q^{\prime} \in Q^{\prime} \cap q M q$, then we write $P \prec_{M}^{s^{\prime}} Q$.

- If both $P \prec_{M}^{s} Q$ and $P \prec_{M}^{s^{\prime}} Q$ hold, then we write $P \prec_{M}^{s, s^{\prime}} Q$.

2.3. From tensor decompositions to group product decompositions. In this subsection we review how some intertwining relations and a tensor product decomposition in a group von Neumann algebra can be used to deduce a direct decomposition of the underlying group, see Theorem 2.3 , The result is actually a mild generalization of [DHI16, Theorem 6.1]. The proof follows the same idea, but for the convenience of the reader we include all the details.

Theorem 2.3 (DHI16]). Let $M=L(\Gamma)$ be the $I I_{1}$ factor of an icc group $\Gamma$ and assume $M=P_{1} \bar{\otimes} P_{2}$. Let $\Sigma_{1}, \Sigma_{2}<\Gamma$ be subgroups and $e \in L\left(\Sigma_{1}\right)^{\prime} \cap M$ a non-zero projection such that

(1) $L\left(\Sigma_{1}\right) e \prec_{M}^{s} P_{1}$ and $P_{1} \prec_{M} L\left(\Sigma_{1}\right) e$,

(2) $L\left(\Sigma_{2}\right) \prec_{M} P_{2}$ and $P_{2} \prec_{M} L\left(\Sigma_{2}\right)$.

Then there exist a decomposition $\Gamma=\Gamma_{1} \times \Gamma_{2}$, a decomposition $M=P_{1}^{t_{1}} \bar{\otimes} P_{2}^{t_{2}}$ for some $t_{1}, t_{2}>0$ with $t_{1} t_{2}=1$ and a unitary $u \in M$ such that $P_{i}^{t_{i}}=u L\left(\Gamma_{i}\right) u^{*}$, for any $i \in \overline{1,2}$.

We first need the following two lemmas inspired by [DHI16, Section 6]. Lemma 2.5 bellow can also be seen as an extension of [Dr19, Lemma 2.6].

Lemma 2.4. Let $M=L(\Gamma)$ be the $I I_{1}$ factor of an icc group $\Gamma$ and assume $M=P_{1} \bar{\otimes} P_{2}$. Let $\Sigma<\Gamma$ be a subgroup and $e \in L(\Sigma)^{\prime} \cap M$ a non-zero projection such that $L(\Sigma) e \prec_{M}^{s} P_{1}$ and $P_{1} \prec_{M} L(\Sigma) e$. Then $Q N_{\Gamma}(\Sigma)<\Gamma$ has finite index.

Proof. We first use [DHI16, Lemma 2.4] and derive that there exists a non-zero projection $f \in$ $\mathcal{Z}\left(L(\Sigma)^{\prime} \cap M\right)$ satisfying $f \leq e$ and

$$
L(\Sigma) f \prec_{M}^{s} P_{1} \text { and } P_{1} \prec_{M}^{s^{\prime}} L(\Sigma) f .
$$

Note that $\mathcal{N}_{M}\left(L(\Sigma)^{\prime} \cap M\right)^{\prime} \cap M \subset L(\Sigma)^{\prime} \cap M$. Hence, by passing to relative commutants in (2.1) it follows from [Va08, Lemma 3.5] and [DHI16, Lemma 2.4] that

$$
P_{2} \prec_{M}^{s^{\prime}}\left(L(\Sigma)^{\prime} \cap M\right) f \text { and }\left(L(\Sigma)^{\prime} \cap M\right) f \prec_{M}^{s} P_{2} .
$$

By repeating the same argument, we deduce that $\left(\left(L(\Sigma)^{\prime} \cap M\right)^{\prime} \cap M\right) f \prec_{M}^{s} P_{1}$. In combination with (2.2) we derive that $\mathcal{Z}\left(\left(L(\Sigma)^{\prime} \cap M\right)\right) f \prec_{M}^{s} P_{1}$ and $\mathcal{Z}\left(\left(L(\Sigma)^{\prime} \cap M\right)\right) f \prec_{M}^{s} P_{2}$. Therefore, by [DHI16, Lemma 2.8(2)] we further obtain that $\mathcal{Z}\left(\left(L(\Sigma)^{\prime} \cap M\right)\right) f \prec_{M}^{s} \mathbb{C} 1$, and hence, there exists a non-zero projection $f_{1} \in \mathcal{Z}\left(\left(L(\Sigma)^{\prime} \cap M\right)\right)$ with $f_{1} \leq f$ such that $\mathcal{Z}\left(\left(L(\Sigma)^{\prime} \cap M\right)\right) f_{1}=\mathbb{C} f_{1}$.

By letting $Q=L(\Sigma) \vee\left(L(\Sigma)^{\prime} \cap M\right)$, it follows that $Q^{\prime} \cap M \subset \mathcal{Z}\left(L(\Sigma)^{\prime} \cap M\right)$, and therefore, $\left(Q f_{1}\right)^{\prime} \cap$ $f_{1} M f_{1}=\mathbb{C} f_{1}$. Using relations (2.1) and (2.2) we have $P_{1} \prec_{M} Q f_{1}$ and $P_{2} \prec_{M} Q f_{1}$. Therefore, we can apply [Dr19, Lemma 2.6] and derive that $M \prec_{M} Q$. Let $\Omega=\left\{g \in \Gamma \mid \mathcal{O}_{\Sigma}(g)\right.$ is finite $\}$, where $\mathcal{O}_{\Sigma}(g)=\left\{h g h^{-1} \mid h \in \Sigma\right\}$ is the orbit of $g$ under conjugation by $\Sigma$. Note that $\Omega$ is normalized by $\Sigma$ and $L(\Sigma)^{\prime} \cap M \subset L(\Omega)$. Since $\Omega \Sigma<\mathrm{QN}_{\Gamma}(\Sigma)$, it follows that $Q \subset L\left(\mathrm{QN}_{\Gamma}(\Sigma)\right)$. Altogether, it follows that $M \prec_{M} L\left(\mathrm{QN}_{\Gamma}(\Sigma)\right)$, which proves the lemma by using [DHI16, Lemma 2.5(1)].

Lemma 2.5. Let $\Gamma \curvearrowright B$ be a trace preserving action and denote $M=B \rtimes \Gamma$. Let $\Sigma_{1}<\Sigma_{2}<\Gamma$ be subgroups such that $L\left(Q N_{\Gamma}\left(\Sigma_{1}\right)\right)^{\prime} \cap M=\mathbb{C} 1$.

Let $P_{1}, P_{2} \subset p M p$ be von Neuman subalgebras such that there exist commuting subalgebras $\tilde{P}_{0}, \tilde{P}_{1}$, $\tilde{P}_{2} \subset M$ satisfying $P_{1} \subset \tilde{P}_{1}, P_{2} \subset \tilde{P}_{2}$ and $\tilde{P}_{0} \vee \tilde{P}_{1} \vee \tilde{P}_{2}=M$.

If $P_{i} \prec_{M} B \rtimes \Sigma_{i}$, for any $i \in \overline{1,2}$, then $P_{1} \vee P_{2} \prec_{M} B \rtimes \Sigma_{2}$. 
Proof. Since $P_{1} \prec_{M} B \rtimes \Sigma_{1}$ and $P_{2} \prec_{M} B \rtimes \Sigma_{2}$, it follows that there exist projections $p_{1} \in P_{1}, p_{2} \in$ $P_{2}$ and non-zero elements $v_{1} \in p_{1} M, v_{2} \in M p_{2}$ such that

$$
\left(p_{1} P_{1} p_{1}\right)_{1} v_{1} \subset v_{1}\left(B \rtimes \Sigma_{1}\right)_{1} \text { and } v_{2}\left(p_{2} P_{2} p_{2}\right)_{1} \subset\left(B \rtimes \Sigma_{2}\right)_{1} v_{2} .
$$

We continue by showing that there exists $g \in Q N_{\Gamma}\left(\Sigma_{1}\right)$ such that $v_{1} u_{g} v_{2} \neq 0$. If this is not the case, we derive that $v_{1} z v_{2}=0$, for all $z \in \mathcal{U}\left(L\left(Q N_{\Gamma}\left(\Sigma_{1}\right)\right)\right)$ and hence, $v_{1} z v_{2} v_{2}^{*} z^{*}=0$. By letting $a=\vee_{z \in \mathcal{U}\left(L\left(Q N_{\Gamma}\left(\Sigma_{1}\right)\right)\right)} z v_{2} v_{2}^{*} z$, it follows that $v_{1} a=0$. Since $a \in L\left(Q N_{\Gamma}\left(\Sigma_{1}\right)\right)^{\prime} \cap M=\mathbb{C} 1$, we derive that $v_{1}=0$ or $v_{2}=0$, contradiction.

Next, we consider $g \in Q N_{\Gamma}\left(\Sigma_{1}\right)$ such that $v_{1} u_{g} v_{2} \neq 0$. This implies that there exist some elements $g_{1}, \ldots, g_{n} \in \Gamma$ such that $\left(B \rtimes \Sigma_{1}\right)_{1} u_{g} \subset \sum_{j=1}^{n} u_{g_{j}}\left(B \rtimes \Sigma_{1}\right)_{1}$. Using (2.3), we derive that

$$
\mathcal{U}\left(p_{1} P_{1} p_{1}\right)\left(v_{1} u_{g} v_{2}\right) \mathcal{U}\left(p_{2} P_{2} p_{2}\right) \subset \sum_{i=1}^{n} v_{1} u_{g_{j}}\left(B \rtimes \Sigma_{2}\right)_{1} v_{2} .
$$

Now, we assume by contradiction that $p_{1} P_{1} p_{1} \vee p_{2} P_{2} p_{2} \nprec_{M} B \rtimes \Sigma_{2}$. Hence, there exist two sequence of unitaries $\left(a_{n}\right)_{n} \subset \mathcal{U}\left(p_{1} P_{1} p_{1}\right)$ and $\left(b_{n}\right)_{n} \subset \mathcal{U}\left(p_{2} P_{2} p_{2}\right)$ such that $\left\|E_{B \rtimes \Sigma_{2}}\left(x a_{n} b_{n} y\right)\right\|_{2} \rightarrow 0$, for all $x, y \in M$. Since $P_{1} \subset \tilde{P}_{1}, P_{2} \subset \tilde{P}_{2}$ and $\tilde{P}_{0} \vee \tilde{P}_{1} \vee \tilde{P}_{2}=M$, we further derive that $\left\|E_{B \rtimes \Sigma_{2}}\left(x a_{n}\left(v_{1} z v_{2}\right) b_{n} y\right)\right\|_{2} \rightarrow 0$, for all $x, y \in M$.

For any subset $F \subset \Gamma$, we denote by $P_{F}$ the orthogonal projection onto the closed linear span of $\left\{B u_{g} \mid g \in F\right\}$ and notice that $\left\|P_{G \Sigma_{2} H}(x)\right\|_{2}^{2} \leq \sum_{g \in G, h \in H}\left\|E_{B \rtimes \Sigma_{2}}\left(u_{g}^{*} x u_{h}^{*}\right)\right\|_{2}^{2}$, for all finite subsets $G, H \subset \Gamma$ and $x \in M$. Hence, the previous paragraph implies that $\left\|P_{G \Sigma_{2} H}\left(a_{n}\left(v_{1} z v_{2}\right) b_{n}\right)\right\|_{2} \rightarrow 0$, for all finite subsets $G, H \subset \Gamma$. By using Kaplansky's density theorem, this contradicts (2.4) and therefore ends the proof of the lemma.

Proof of Theorem [2.3. From assumption we have $L\left(\Sigma_{1}\right) \prec_{M} L\left(\Sigma_{2}\right)^{\prime} \cap M$. By applying [DHI16, Theorem 6.2] we obtain that there exist finite index subgroups $\Theta_{1}<k \Sigma_{1} k^{-1}$ and $\Theta_{2}<\Sigma_{2}$ for some $k \in \Gamma$ such that the commutator subgroup $\left[\Theta_{1}, \Theta_{2}\right]$ is finite. Denote by $\Theta<\Gamma$ the subgroup generated by $\Theta_{1}$ and $\Theta_{2}$.

By Lemma 2.4 we have $\left[\Gamma: \mathrm{QN}_{\Gamma}\left(\Sigma_{1}\right)\right]<\infty$. Since $\Gamma$ is icc, we derive that $L\left(\mathrm{QN}_{\Gamma}\left(\Theta_{1}\right)\right)^{\prime} \cap M=\mathbb{C} 1$. Therefore, by Lemma 2.5 we derive that $M \prec_{M} L(\Theta)$, which shows that $[\Gamma: \Theta]<\infty$. In particular, $\Theta$ is icc. Since $\left[\Theta_{1}, \Theta_{2}\right]$ is a finite normal subgroup of $\Theta$, it must be trivial. Therefore, $\Theta_{1}$ and $\Theta_{2}$ are commuting subgroups of $\Gamma$ such that $\Theta_{1} \cap \Theta_{2}=1$ and $\left[\Gamma: \Theta_{1} \Theta_{2}\right]<\infty$.

Finally, by applying verbatim the second part of the proof of [DHI16, Theorem 6.1] (or the second part of the proof of [CdSS15, Theorem 4.14]), we obtain the conclusion of the theorem.

2.4. An augmentation technique for intertwining. An essential tool for the proof of our main result, Theorem A, is the augmentation technique developed in CD-AD20; this was also crucial for the recent work CD-AD21. One place where the augmentation technique is used in our paper is in the following result.

Proposition 2.6. Let $\Gamma$ be a countable icc group and let $M=L(\Gamma)$. Let $\Sigma, \Theta<\Gamma$ be subgroups and $M=P \bar{\otimes} Q$ a tensor product decomposition. Let $G_{0}<G$ be countable groups and let $G \curvearrowright A$ be a trace preserving action such that $Q=A \rtimes G$. Assume that the following two conditions hold:

- $P \prec_{M} L(\Sigma)$ and $L(\Sigma) \prec_{M} P$.

- $Q \prec_{M} L(\Theta)$ and $L(\Theta) \prec_{M} P \bar{\otimes}\left(A \rtimes G_{0}\right)$.

Then $\left[G: G_{0}\right]<\infty$. 
Proof. Following the augmentation technique from [CD-AD20, Section 3], we consider a Bernoulli action $\Gamma \curvearrowright D$ with abelian base. Denote $\mathcal{M}=D \rtimes \Gamma$ and let $\Psi: \mathcal{M} \rightarrow \mathcal{M} \bar{\otimes} M$ be the *homomorphism given by $\Psi\left(d u_{g}\right)=d u_{g} \otimes u_{g}$, for all $d \in D$ and $g \in \Gamma$.

From [DHI16, Lemma 2.4(2)] we get that $P \prec_{M}^{s} L(\Sigma)$, and hence, $P \prec_{\mathcal{M}}^{s, s^{\prime}} D \rtimes \Sigma$. By using [DHI16, Remark 2.2] and [Dr19, Lemma 2.3] we further obtain that $\Psi(P) \prec_{\mathcal{M} \bar{\otimes} M}^{s, s^{\prime}} \mathcal{M} \bar{\otimes} L(\Sigma)$. Using $L(\Sigma) \prec_{M} P$ and [Dr19, Lemma 2.4(2)], we get $\Psi(P) \prec_{\mathcal{M} \bar{\otimes} M}^{s} \mathcal{M} \bar{\otimes} P$. In the same way, we derive that $\Psi(Q) \prec_{\mathcal{M} \bar{\otimes} M}^{s} \mathcal{M} \bar{\otimes} P \bar{\otimes}\left(A \rtimes G_{0}\right)$. Using [BV12, Lemma 2.3] we get that $\Psi(M) \prec_{\mathcal{M} \bar{\otimes} M} \mathcal{M} \bar{\otimes} P \bar{\otimes}(A \rtimes$ $\left.G_{0}\right)$. Since $\Psi(x)=x \otimes 1$, for all $x \in D$, we deduce that $\Psi(\mathcal{M}) \prec_{\mathcal{M}} \bar{\otimes} M \mathcal{M} \bar{\otimes} P \bar{\otimes}\left(A \rtimes G_{0}\right)$. By [IPV10, Lemma 10.2] we obtain that $M \prec \mathcal{M} P \bar{\otimes}\left(A \rtimes G_{0}\right)$. Since $\mathcal{Q} \mathcal{N}_{\mathcal{M}}^{(1)}(M)=M$, we derive that $M \prec_{M} P \bar{\otimes}\left(A \rtimes G_{0}\right)$, which implies from [DHI16, Lemma 2.5(1)] that $\left[G: G_{0}\right]<\infty$.

2.5. Relative amenability and weak containment of bimodules. A tracial von Neumann algebra $(M, \tau)$ is amenable if there exists a positive linear functional $\Phi: \mathbb{B}\left(L^{2}(M)\right) \rightarrow \mathbb{C}$ such that $\Phi_{\mid M}=\tau$ and $\Phi$ is $M$-central, meaning $\Phi(x T)=\Phi(T x)$, for all $x \in M$ and $T \in \mathbb{B}\left(L^{2}(M)\right)$. By Connes' breakthrough classification of amenable factors [Co76], it follows that $M$ is amenable if and only if $M$ is approximately finite dimensional.

Next, we recall the notion of relative amenability introduced by Ozawa and Popa in [OP07. Let $(M, \tau)$ be a tracial von Neumann algebra. Let $p \in M$ be a projection and $P \subset p M p, Q \subset M$ be von Neumann subalgebras. Following [P07, Definition 2.2], we say that $P$ is amenable relative to $Q$ inside $M$ if there exists a positive linear functional $\Phi: p\left\langle M, e_{Q}\right\rangle p \rightarrow \mathbb{C}$ such that $\Phi_{\mid p M p}=\tau$ and $\Phi$ is $P$-central. Note that $P$ is amenable relative to $\mathbb{C}$ inside $M$ if and only if $P$ is amenable. We say that $P$ is strongly non-amenable relative to $Q$ if $P p^{\prime}$ is non-amenable relative to $Q$ for any non-zero projection $p^{\prime} \in P^{\prime} \cap p M p$.

Let $M, N$ be tracial von Neumann algebras. An $M-N$ bimodule ${ }_{M} \mathcal{H}_{N}$ is a Hilbert space $\mathcal{H}$ equipped with a $*$-homomorphism $\pi_{\mathcal{H}}: M \odot N^{\mathrm{op}} \rightarrow \mathbb{B}(\mathcal{H})$ that is normal on $M$ and $N^{\mathrm{op}}$, where $M \odot N^{\mathrm{op}}$ is the algebraic tensor product between $M$ and the oposite von Neumann algebra $N^{\text {op }}$ of $N$. For two $M-N$ bimodules ${ }_{M} \mathcal{H}_{N}$ and ${ }_{M} \mathcal{K}_{N}$, we say that ${ }_{M} \mathcal{H}_{N}$ is weakly contained in ${ }_{M} \mathcal{K}_{N}$ if $\left\|\pi_{\mathcal{H}}(x)\right\| \leq\left\|\pi_{\mathcal{K}}(x)\right\|$, for any $x \in M \odot N^{\mathrm{op}}$. Examples of bimodules include the trivial bimodule ${ }_{M} L^{2}(M)_{M}$ and the coarse bimodule ${ }_{M} L^{2}(M) \otimes L^{2}(N)_{N}$.

Finally, if $Q \subset M$ are tracial von Neumann algebras, then ${ }_{M} L^{2}\left(\left\langle M, e_{Q}\right\rangle\right)_{M} \cong_{M}\left(L^{2}(M) \otimes_{Q} L^{2}(M)\right)_{M}$. Also, a subalgebra $P \subset p M p$ is amenable relative to $Q$ if and only if ${ }_{P} L^{2}(p M)_{M}$ is weakly contained in ${ }_{P} L^{2}\left(\left\langle M, e_{Q}\right\rangle\right)_{M}$.

2.6. Mixing inclusion of von Neumann algebras. An important tool in the intertwining-bybimodules techniques is the mixing notion relative to a subalgebra (see [Po05, Definition 2.9], [PS09, Definition 2.3] and [Bo14, Definition A.4.2]).

Definition 2.7. Let $A \subset M \subset \tilde{M}$ be an inclusion of tracial von Neumann algebras. We say that $M \subset \tilde{M}$ is mixing relative to $A$ if for any sequence $\left(u_{n}\right)_{n} \subset(M)_{1}$ satisfying $\left\|E_{A}\left(x u_{n} y\right)\right\|_{2} \rightarrow 0$, for all $x, y \in M$, we have $\left\|E_{M}\left(\tilde{x} u_{n} \tilde{y}\right)\right\|_{2} \rightarrow 0$, for all $\tilde{x}, \tilde{y} \in \tilde{M} \ominus M$.

In Definition 2.7, if $A=\mathbb{C} 1$ we simply say that $M \subset \tilde{M}$ is mixing. We record the following well known lemmas and include the proof of the first one only for the convenience of the reader; the second one can be proven in a similar way.

Lemma 2.8. Let $\Sigma<\Gamma$ be countable groups and denote $I=\Gamma / \Sigma$. Let $A_{0} \subset B_{0}$ be tracial von Neumann algebras and denote $M=A_{0}^{I} \rtimes \Gamma$ and $\tilde{M}=B_{0}^{I} \rtimes \Gamma$. Note that $M \subset \tilde{M}$.

Then $M \subset \tilde{M}$ is mixing relative to $A_{0}^{I} \rtimes \Sigma$. 
Proof. Let $\left(w_{n}\right)_{n} \subset \mathcal{U}(M)$ be a sequence of unitaries such that $\left\|E_{A_{0}^{I} \rtimes \Sigma}\left(x w_{n} y\right)\right\|_{2} \rightarrow 0$, for all $x, y \in M$. We have to show that $\left\|E_{M}\left(\tilde{x} w_{n} \tilde{y}\right)\right\|_{2} \rightarrow 0$, for all $\tilde{x}, \tilde{y} \in \tilde{M} \ominus M$. By Kaplansky's density theorem, it is enough to assume $\tilde{x}=b \in\left(B_{0} \ominus A_{0}\right)^{h \Sigma}$ and $\tilde{y}=c \in\left(B_{0} \ominus A_{0}\right)^{k \Sigma}$ for some $h, k \in I$. If we let $w_{n}=\sum_{g \in \Gamma} w_{n}^{g} u_{g} \in A_{0}^{I} \rtimes \Gamma$, note that $E_{M}\left(b w_{n}^{g} \sigma_{g}(c)\right)=0$ if $g \notin h \Sigma k^{-1}$ and hence

$$
\left\|E_{M}\left(\tilde{x} w_{n} \tilde{y}\right)\right\|_{2} \leq\|b\|\|c\|\left\|\sum_{g \in h \Sigma k^{-1}} w_{n}^{g} u_{g}\right\|_{2}=\|b\|\|c\|\left\|E_{A_{0}^{I} \rtimes \Sigma}\left(u_{h^{-1}} w_{n} u_{k}\right)\right\|_{2} \rightarrow 0,
$$

by the assumption.

Lemma 2.9. Let $\tilde{M}=M_{1} *_{A} M_{2}$ be an amalgamated free product of tracial von Neumann algebras. Then $M_{1} \subset \tilde{M}$ is mixing relative to $A$.

\section{Two Classes of von Neumann algebras that admit S-Malleable Deformations}

3.1. Malleable deformations. In $\mathrm{Po01}, \mathrm{Po03}$ Popa introduced the notion of an s-malleable deformation of a von Neumann algebra. In the framework of his powerful deformation/rigidity techniques, this notion has led to a remarkable progress in the theory of von Neumann algebras, see the surveys [Po07,Va10a, Io12b, Io17]. See also dSHHS20] for a comprehensive overview on s-malleable deformations and for recent developments.

Definition 3.1. Let $(M, \tau)$ be a tracial von Neumannn algebra. A pair $\left(\tilde{M},\left(\alpha_{t}\right)_{t \in \mathbb{R}}\right)$ is called an s-malleable deformation of $M$ if the following conditions hold:

- $(\tilde{M}, \tilde{\tau})$ is a tracial von Neumann algebra such that $M \subset \tilde{M}$ and $\tau=\tilde{\tau}_{\mid M}$.

- $\left(\alpha_{t}\right)_{t \in \mathbb{R}} \subset \operatorname{Aut}(\tilde{M}, \tilde{\tau})$ is a 1-parameter group with $\lim _{t \rightarrow 0}\left\|\alpha_{t}(x)-x\right\|_{2}=0$, for any $x \in \tilde{M}$.

- There exists $\beta \in \operatorname{Aut}(\tilde{M}, \tilde{\tau})$ that satisfies $\beta_{\mid M}=\operatorname{Id}_{M}, \beta^{2}=\operatorname{Id}_{\tilde{M}}$ and $\beta \alpha_{t}=\alpha_{-t} \beta$, for any $t \in \mathbb{R}$.

- $\alpha_{t}$ does not converge uniformly to the identity on $(M)_{1}$ as $t \rightarrow 0$.

Theorem 3.2 ([dSHHS20]). Let $\left(\tilde{M},\left(\alpha_{t}\right)_{t \in \mathbb{R}}\right)$ be an s-malleable deformation of a tracial von Neumann algebra $M$. Let $A \subset M$ and $Q \subset q M q$ be some von Neumann subalgebras such that $M \subset \tilde{M}$ is mixing relative to $A, \alpha_{t} \rightarrow$ id uniformly on $(Q)_{1}$ and $Q \nprec_{M} A$. Then the following hold:

(1) $\alpha_{t} \rightarrow$ id uniformly on $\left(Q \vee\left(Q^{\prime} \cap q M q\right)\right)_{1}$.

(2) Let $Q=Q_{1} \subset Q_{2} \subset \cdots \subset q M q$ be an ascending sequence of von Neumann subalgebras with $\alpha_{t} \rightarrow$ id uniformly on $\left(Q_{i}\right)_{1}$, for all $i \geq 1$. Then $\alpha_{t} \rightarrow$ id uniformly on $\left(\bigvee_{i \geq 1} Q_{i}\right)_{1}$.

Proof. Part (1) follows from dSHHS20, Corollary 6.7(ii)]. For part (2), by using dSHHS20, Lemma 6.3] it follows that $Q^{\prime} \cap q \tilde{M} q \subset q M q$ and the conclusion follows from dSHHS20, Theorem 3.5].

We will also need the following result from dSHHS20, Proposition 5.6].

Proposition 3.3 ( dSHHS20]). Let $\left(\tilde{M},\left(\alpha_{t}\right)_{t \in \mathbb{R}}\right)$ be an s-malleable deformation of a tracial von Neumann algebra $M$. Let $Q \subset q M q$ be a von Neumann subalgebra and let $q_{0} \in Q$ be a non-zero projection such that $\alpha_{t} \rightarrow$ id uniformly on $\left(q_{0} Q q_{0}\right)_{1}$.

Then $\alpha_{t} \rightarrow$ id uniformly on $(Q z)_{1}$, where $z$ is the central support of $q_{0}$ in $Q$.

3.2. Class $\mathcal{M}$. We say that a non-amenable $\mathrm{II}_{1}$ factor $M$ is in Class $\mathcal{M}$ if there exists an s-malleable deformation $\left(\tilde{M},\left(\alpha_{t}\right)_{t \in \mathbb{R}}\right)$ of $M$ and an amenable subalgebra $A \subset M$ satisfying:

(1) The inclusion $M \subset \tilde{M}$ is mixing relative to $A$.

(2) For any tracial von Neumann algebra $N$ and for any subalgebra $P \subset p(M \bar{\otimes} N) p$ such that $P^{\prime} \cap p(M \bar{\otimes} N) p$ is strongly non-amenable relative to $1 \otimes N$, it follows that: 
(i) $\alpha_{t} \otimes$ id $\rightarrow$ id uniformly on $(P)_{1}$.

(ii) If $P \prec_{M \otimes N} A \otimes N$, then $P \prec_{M \otimes N} 1 \otimes N$.

While this class of $\mathrm{II}_{1}$ factors seems somewhat technical, it actually contains all group von Neumann algebras $L(\Gamma)$ with $\Gamma \in \mathcal{A}$ and all non-trivial tracial free products $M_{1} * M_{2}$, see Proposition 3.4 bellow and its proof. Note also that if $A=\mathbb{C} 1$, then condition (2) is simply reflecting Popa's spectral gap principle.

Proposition 3.4. If $\Gamma \in \mathcal{A}$, then $L(\Gamma) \in \mathcal{M}$.

Proof. If $\Gamma \in \mathcal{A}_{1}$ we recall that Sinclair constructed in [Si10, Section 3] an s-malleable deformation $\left(\tilde{M},\left(\alpha_{t}\right)_{t \in \mathbb{R}}\right)$ in the sense of Definition 3.1; see also [Va10b, Section 3.1] and [Io11, Section 2]. We will prove that $M \in \mathcal{M}$ with $A=\mathbb{C} 1$. Note first that $M \subset \tilde{M}$ is mixing since $\pi$ is a mixing representation. Next, Lemma 3.5 bellow shows that condition (2) of Class $\mathcal{M}$ is satisfied.

Next, if $\Gamma=\Gamma_{1} *_{\Sigma} \Gamma_{2} \in \mathcal{A}_{2}$, we recall that IPP05, Section 2.2] shows that $M=L(\Gamma)$ admits an smalleable deformation $\left(\tilde{M},\left(\alpha_{t}\right)_{t \in \mathbb{R}}\right)$ in the sense of Definition 3.1 with $\tilde{M}=M *_{L(\Sigma)}\left(L(\Sigma) \bar{\otimes} L\left(\mathbb{F}_{2}\right)\right)$. It follows that $M \in \mathcal{M}$ with $A=L(\Sigma)$. Indeed, we note first that $M \subset \tilde{M}$ is mixing relative to $A$ by Lemma 2.9. Next, since we have the decomposition $M \bar{\otimes} N=\left(L\left(\Gamma_{1}\right) \bar{\otimes} N\right) *_{L}(\Sigma) \bar{\otimes} N\left(L\left(\Gamma_{2}\right) \bar{\otimes} N\right)$, condition (2.i) follows from [Io12a, Lemma 6.5]. To show condition (2.ii), let $N$ be a tracial von Neumann algebra and $P \subset p(M \bar{\otimes} N) p$ a subalgebra such that $P^{\prime} \cap p(M \bar{\otimes} N) p$ is strongly nonamenable relative to $1 \otimes N$. If $P \prec_{M \otimes N} A \otimes N$ and $P \nprec_{M \otimes N} 1 \otimes N$, then we derive (see, e.g., Dr17, Proposition 3.7]) that $P^{\prime} \cap p(M \bar{\otimes} N) p \prec_{M \bar{\otimes} N} A \bar{\otimes} N$. It implies by [DHI16, Lemma $2.6(3)]$ that $P^{\prime} \cap p(M \bar{\otimes} N) p$ is not strongly non-amenable relative to $1 \otimes N$, contradiction.

Finally, if $\Gamma \in \mathcal{A}_{3}$, we recall that Ioana constructed in [I006, Section 2] an s-malleable deformation $\left(\tilde{M},\left(\alpha_{t}\right)_{t \in \mathbb{R}}\right)$ in the sense of Definition 3.1, see Remark 3.8(1). Next, we note that $M \subset \tilde{M}$ is mixing relative to $A:=L(\Sigma)^{G / H} \rtimes H$ by Lemma 2.8. Next, parts (i) and (ii) from condition (2) of Class $\mathcal{M}$ follow from [IPV10, Corollary 4.3] and its proof.

The following lemma is a standard application of Popa's spectral gap rigidity principle $\mathrm{Po06 \textrm {b }}$, and it is essentially contained in the proof of [Ho15, Theorem 6.4] (see also [Io11, Lemma 2.2]). For completeness, we include a proof.

Lemma 3.5. Let $\Gamma$ be a countable non-amenable group that admits an unbounded cocycle for some mixing representation $\pi: \Gamma \rightarrow \mathcal{O}\left(H_{\mathbb{R}}\right)$ such that $\pi$ is weakly contained in the left regular representation of $\Gamma$. Let $\Gamma \curvearrowright N$ be a trace preserving action and denote $M=N \rtimes \Gamma$.

If $P \subset p M p$ is a von Neumann subalgebra that is strongly non-amenable relative to $N$, then $\alpha_{t} \rightarrow i d$ uniformly on $\left(P^{\prime} \cap p M p\right)_{1}$.

Proof. Since $\pi$ is contained in the left regular representation, it follows by Va10b, Lemma 3.5] that the $M-M$ bimodule $L^{2}(\tilde{M} \ominus M)$ is weakly contained in the $M$ - $M$ bimodule $L^{2}(M) \otimes_{N} L^{2}(M)$. Since $P$ is strongly non-amenable relative to $N$, it follows that for any non-zero projection $z \in$ $\mathcal{Z}\left(P^{\prime} \cap p M p\right)$ we have that ${ }_{M} L^{2}(M)_{P z}$ is not weakly contained in ${ }_{M} L^{2}(M) \otimes_{N} L^{2}(M)_{P z}$. Hence, for any non-zero projection $z \in \mathcal{Z}\left(P^{\prime} \cap p M p\right)$ we derive that ${ }_{M} L^{2}(M)_{P z}$ is not weakly contained in ${ }_{M} L^{2}(\tilde{M} \ominus M)_{P z}$.

Let $\epsilon>0$. Therefore, by [IPV10, Lemma 2.3] we obtain that there exist $a_{1}, \ldots, a_{n} \in P$ and $\delta>0$ such that if $x \in(p \tilde{M} p)_{1}$ satisfies $\left\|x a_{i}-a_{i} x\right\|_{2} \leq \delta$, for any $i \in \overline{1, n}$, then $\left\|x-E_{M}(x)\right\|_{2} \leq \epsilon$. We choose $t_{0}>0$ such that $\left\|\alpha_{t}\left(a_{i}\right)-a_{i}\right\|_{2} \leq \delta / 2$, for all $|t| \leq t_{0}$ and $i \in \overline{1, n}$. Let $x \in\left(P^{\prime} \cap p M p\right)_{1}$ and $t \in \mathbb{R}$ such that $|t| \leq t_{0}$. By using the triangle inequality we derive that for all $i \in \overline{1, n}$ and $|t| \leq t_{0}$ we have

$$
\left\|a_{i} \alpha_{t}(x)-\alpha_{t}(x) a_{i}\right\|_{2}=\left\|\alpha_{-t}\left(a_{i}\right) x-x \alpha_{-t}\left(a_{i}\right)\right\|_{2} \leq 2\left\|\alpha_{-t}\left(a_{i}\right)-a_{i}\right\|_{2} \leq \delta .
$$


As a consequence, we deduce that $\left\|\alpha_{t}(x)-E_{M}\left(\alpha_{t}(x)\right)\right\|_{2} \leq \epsilon$, for any $|t| \leq t_{0}$. Using Popa's transversality property [Va10b, Lemma 3.1] we obtain that

$$
\left\|\alpha_{t}(x)-x\right\|_{2} \leq \sqrt{2} \epsilon \text {, for all } x \in\left(P^{\prime} \cap p M p\right)_{1} \text { and }|t| \leq t_{0} .
$$

This concludes the proof.

Remark 3.6. The proof of Lemma 3.5 shows that the class $\mathcal{M}_{0}$ defined in Remark 1.1 is contained in $\mathcal{M}$ and that any non-amenable tracial free product $M_{1} * M_{2}$ belongs to $\mathcal{M}_{0}$ (see also Io12a, Lemma 2.10]).

We end this subsection by showing that any von Neumann algebra $M \in \mathcal{M}$ does not have property Gamma, i.e. for any uniformly bounded sequence $\left(x_{n}\right)_{n} \subset M$ with $\left\|x_{n} y-y x_{n}\right\|_{2} \rightarrow 0$, for any $y \in M$, must satisfy $\left\|x_{n}-\tau\left(x_{n}\right)\right\|_{2} \rightarrow 0$.

Lemma 3.7. Let $M$ be a tracial von Neumann algebra that belongs to Class $\mathcal{M}$. Then $M$ does not have property Gamma.

Proof. We assume the contrary. By using [HU15, Theorem 3.1] it follows that there exists a decreasing sequence of diffuse abelian von Neumann subalgebras $A_{n} \subset M$ with $n \geq 1$ such that $M=\bigvee_{n \geq 1}\left(A_{n}^{\prime} \cap M\right)$.

Let $\left(\tilde{M},\left(\alpha_{t}\right)_{t \in \mathbb{R}}\right)$ be an s-malleable deformation of $M$ and $A \subset M$ a subalgebra as given by the definition of Class $\mathcal{M}$. Since $M$ is non-amenable, it follows that there exists $n \geq 1$ such that $A_{n}^{\prime} \cap M$ is non-amenable. Let $z \in \mathcal{Z}\left(A_{n}^{\prime} \cap M\right)$ such that $\left(A_{n}^{\prime} \cap M\right) z$ is strongly non-amenable relative to $\mathbb{C} 1$. By using the fact that $M$ belongs to Class $\mathcal{M}$, it follows that $\alpha_{t} \rightarrow$ id uniformly on $\left(A_{n} z\right)_{1}$. In particular,

$$
\alpha_{t} \rightarrow \text { id uniformly on }\left(A_{m} z\right)_{1} \text {, for any } m \geq n \text {. }
$$

Note here that $z \in A_{m}^{\prime} \cap M$, for any $m \geq n$. If $A_{m} z \prec_{M} A$, condition (2.ii) of Class $\mathcal{M}$ shows that there exists a non-zero projection $z_{1} \in\left(A_{m}^{\prime} \cap M\right)^{\prime} \cap M \subset\left(A_{n}^{\prime} \cap M\right)^{\prime} \cap M$ with $z_{1} \leq z$ such that $\left(A_{m}^{\prime} \cap M\right) z_{1}$ is amenable. This proves that $\left(A_{n}^{\prime} \cap M\right) z_{1}$ is amenable, contradiction.

Hence, $A_{m} z \nprec_{M} A$. Since $M \subset \tilde{M}$ is mixing relative to $A$, it follows from (3.1) and Theorem 3.2(1) that $\alpha_{t} \rightarrow$ id uniformly on $\left(z\left(A_{m}^{\prime} \cap M\right) z\right)_{1}$, for any $m \geq n$. Since $z\left(A_{m}^{\prime} \cap M\right) z \nprec_{M} A$ and $z M z=\bigvee_{m>n} z\left(A_{m}^{\prime} \cap M\right) z$, we apply Theorem 3.2(2) and derive that $\alpha_{t} \rightarrow$ id uniformly on $(z M z)_{1}$. Since $\bar{M}$ is a factor, we apply Proposition 3.3 and deduce that $\alpha_{t} \rightarrow$ id uniformly on $(M)_{1}$, contradiction. Therefore, $M$ does not have property Gamma.

3.3. Class $\mathcal{M}_{w r}$. We say that a von Neumann algebra $M$ is in Class $\mathcal{M}_{w r}$ if there exists a decomposition $M=B_{0}^{I} \rtimes \Lambda$ satisfying the following properties:

- $B_{0}$ is a tracial amenable von Neumann algebra and $\Lambda$ is a non-amenable group.

- There exists $k \geq 1$ such that Stab $J$ is finite whenever $J \subset I$ with $|J| \geq k$.

Remark 3.8. We record the following properties of von Neumann algebras that belong to $\mathcal{M}_{w r}$.

(1) Throughout the proofs of the main results, we will use the fact that any $M \in \mathcal{M}_{w r}$ admits an s-malleable deformation $\left(\tilde{M},\left(\alpha_{t}\right)_{t \in \mathbb{R}}\right)$ in the sense of Definition 3.1 by using the free product deformation, see [Io06, Section 2]. To recall this construction, we define a self-adjoint unitary $h \in L(\mathbb{Z})$ with spectrum $[-\pi, \pi]$ such that $\exp (i h)$ equals the canonical generating unitary $u \in L(\mathbb{Z})$. For any $t \in \mathbb{R}$, define $u_{t}=\exp (i t h) \in L(\mathbb{Z})$. We let $\tilde{M}=L\left(B_{0} * L(\mathbb{Z})\right)^{I} \rtimes \Lambda \supset M$ and $\alpha_{t}=\otimes_{i \in I} \operatorname{Ad}\left(u_{t}\right) \in \operatorname{Aut}(\tilde{M})$.

(2) If $M \in \mathcal{M}_{w r}$, then $M$ is a $\mathrm{II}_{1}$ factor without property Gamma (see, e.g., Dr20, Proposition $4.3])$. 


\section{From commuting subalgebras to Commuting Subgroups}

One of the crucial ingredients of the proof of Theorem $\mathrm{A}$ is an ultrapower technique due to Adrian Ioana [Io11, which we recall in the following form. This result is essentially contained in the proof of [Io11, Theorem 3.1] (see also [CdSS15, Theorem 3.3]). The statement that we will use is a particular case of [DHI16, Theorem 4.1].

Theorem 4.1 (【Io11]). Let $\Gamma$ be a countable icc group and denote by $M=L(\Gamma)$. Let $\Delta: M \rightarrow$ $M \bar{\otimes} M$ be the *-homomorphism given by $\Delta\left(u_{g}\right)=u_{g} \otimes u_{g}$, for all $g \in \Gamma$. Let $B, Q \subset M$ be von Neumann subalgebras such that $\Delta(B) \prec_{M \bar{\otimes} M} M \bar{\otimes} Q$.

Then there exists a decreasing sequence of subgroups $\Sigma_{k}<\Gamma$ such that $B \prec_{M} L\left(\Sigma_{k}\right)$, for every $k \geq 1$, and $Q^{\prime} \cap M \prec_{M} L\left(\cup_{k \geq 1} C_{\Gamma}\left(\Sigma_{k}\right)\right)$.

Note that the ultrapower technique [Io11] has been of crucial use in several other works in order to obtain structural and rigidity results for certain classes of group and group measure space von Neumann algebras [CdSS15, KV15, DHI16, CI17, CU18, Dr19, CDK19, CDHK20, CD-AD20, CD-AD21].

By combining Theorem 4.1 with a recent characterization of von Neumann algebras that do not have property Gamma [BMO19, IM19] we obtain the following useful consequence.

Theorem 4.2. We consider the context of Theorem 4.1. In addition, assume that $M=P \bar{\otimes} Q$ where $P$ does not have property Gamma.

Then there exists a subgroup $\Sigma<\Gamma$ with non-amenable centralizer $C_{\Gamma}(\Sigma)$ such that $B \prec_{M} L(\Sigma)$ and $L(\Sigma) \prec_{M} Q$.

Proof. The proof is inspired by [IM19, Lemma 5.2]. We start the proof by recalling two general facts from [BMO19]. First, BMO19, Proposition 3.2] is stating that if a tracial von Neumann algebra $N$ does not have property Gamma, then any $N-N$ bimodule $\mathcal{K}$ that is weakly equivalent to $L^{2}(N)$ (i.e. ${ }_{N} \mathcal{K}_{N}$ is weakly contained in $L^{2}(N)$ and $L^{2}(N)$ is weakly contained in ${ }_{N} \mathcal{K}_{N}$ ) must contain $L^{2}(N)$.

Second, by using [BMO19, Lemma 3.4] we derive that for any subalgebra $Q_{0} \subset M$ we have

$$
L^{2}\left(\left\langle M, e_{Q_{0}^{\prime} \cap M}\right\rangle\right) \text { is weakly contained in } L^{2}(M) \text { as } M-M \text { bimodules. }
$$

Next, we consider a decreasing sequence of subgroups $\Sigma_{k}<\Gamma$ as in the conclusion of Theorem 4.1, Since $P$ is non-amenable, we can assume without loss of generality that $C_{\Gamma}\left(\Sigma_{k}\right)$ is non-amenable for any $k \geq 1$. Denote the $M-M$ bimodule $\mathcal{H}=\oplus_{k \geq 1} L^{2}\left(\left\langle M, e_{L\left(\Sigma_{k}\right)^{\prime} \cap M}\right\rangle\right)$ and notice that (4.1) implies that

$\mathcal{H}$ is weakly contained in $L^{2}(M)$ as $M-M$ bimodules.

On the other hand, since $P \prec_{M} L\left(\cup_{k \geq 1} C_{\Gamma}\left(\Sigma_{k}\right)\right)$, it follows by Lemma 2.4(2) and Lemma 2.6(3) from [DHI16] that $P$ is amenable relative to $\bigvee_{k \geq 1}\left(L\left(\Sigma_{k}\right)^{\prime} \cap M\right)$ inside $M$. Hence, $L^{2}(M)$ is weakly contained in $L^{2}\left(\left\langle M, e \bigvee_{k \geq 1}\left(L\left(\Sigma_{k}\right)^{\prime} \cap M\right)\right\rangle\right)$ as $M-P$ bimodules. By using the moreover part of [IM19, Proposition 2.5], we deduce that $L^{2}\left(\left\langle M, e \bigvee_{k \geq 1}\left(L\left(\Sigma_{k}\right)^{\prime} \cap M\right)\right\rangle\right)$ is weakly contained in $\mathcal{H}$ as $M-M$ bimodules. Therefore,

$$
L^{2}(M) \text { is weakly contained in } \mathcal{H} \text { as } M-P \text { bimodules. }
$$

Since $M=P \bar{\otimes} Q$, it follows that $L^{2}(M)$ is a multiple of $L^{2}(P)$ as $P-P$ bimodules. In combination with (4.2) and (4.3), we deduce that $L^{2}(P)$ is weakly equivalent to $\mathcal{H}$ as $P-P$ bimodules. Finally, since $P$ does not have property Gamma, we use the first paragraph of the proof and derive that $L^{2}(P)$ is contained in ${ }_{P} \mathcal{H}_{P}$. Hence, there exists $k_{0} \geq 1$ such that $L^{2}(P)$ is contained in 
${ }_{P} L^{2}\left(M, e_{L\left(\Sigma_{k_{0}}\right)^{\prime} \cap M}\right)_{P}$. This implies that $P \prec_{M} L\left(\Sigma_{k_{0}}\right)^{\prime} \cap M$. By passing to relative commutants and using [Va08, Lemma 3.5], the conclusion of the corollary is obtained by taking $\Sigma=\Sigma_{k_{0}}$.

As an application of Theorem 4.2, we can use the augmentation technique from [CD-AD20, Section $3]$ and derive the following result which is an important ingredient of the proof of Theorem A.

Theorem 4.3. Let $\Gamma$ be a countable icc group such that $L(\Gamma)$ does not have property Gamma. Denote $M=L(\Gamma)^{1 / t}$ for some $t>0$ and let $\Delta: M^{t} \rightarrow M^{t} \bar{\otimes} M^{t}$ be the $*$-homomorphism given by $\Delta\left(u_{g}\right)=u_{g} \otimes u_{g}$, for any $g \in \Gamma$.

Assume $M=M_{1} \bar{\otimes} \ldots \bar{\otimes} M_{n}$ is the tensor product of $n \geq 1 I I_{1}$ factors with the property that for any $i \in \overline{1, n}$, there exists $f(i) \in \overline{1, n}$ such that $\Delta\left(M_{\hat{i}}^{t}\right) \prec_{M^{t} \bar{\otimes} M^{t}}^{s} M^{t} \bar{\otimes} M_{\overline{f(i)}}^{t}$.

Then $\Delta\left(M_{I}^{t}\right) \prec_{M^{t} \bar{\otimes} M^{t}}^{s} M^{t} \bar{\otimes} M_{I}^{t}$, for any subset $I \subset \overline{1, n}$.

Proof. We may assume that $t=1$ since this simplification does not hide any essential part of the argument. Indeed, note that for any $i \in \overline{1, n}$ there is a natural identification $M^{t}=M_{\bar{i}} \bar{\otimes} M_{i}^{t}$. Assume by contradiction that there exists $i$ such that $f(i) \neq i$. Using the assumption we can apply Theorem 4.2 and derive that for any $j \in \overline{1, n}$ there exists a subgroup $\Sigma_{j}<\Gamma$ such that

$$
M_{\widehat{j}} \prec_{M}^{s} L\left(\Sigma_{j}\right) \text { and } L\left(\Sigma_{j}\right) \prec_{M} M_{\widehat{f(j)}} .
$$

Here, we also used [DHI16, Lemma 2.4(2)]. Note that (4.4) shows that $L\left(\Sigma_{i}\right) \prec_{M} M_{\widehat{f(i)}}$ and $M_{\widehat{f(i)}} \prec_{M}^{s} L\left(\Sigma_{f(i)}\right)$. Using [Va08, Lemma 3.7] we deduce that $L\left(\Sigma_{i}\right) \prec_{M} L\left(\Sigma_{f(i)}\right)$. We can apply [CI17, Lemma 2.2] and derive that there exist $g \in \Gamma$ and a finite index subgroup $\Sigma_{i}^{0}<\Sigma_{i}$ such that $g \Sigma_{i}^{0} g^{-1} \subset \Sigma_{f(i)}$. Using (4.4) for $j=f(i)$ we get that $L\left(\Sigma_{i}^{0}\right) \prec_{M} M_{\widehat{f^{2}(i)}}$. Since $\left[\Sigma_{i}: \Sigma_{i}^{0}\right]<\infty$, it follows that

$$
L\left(\Sigma_{i}\right) \prec_{M} M_{\widehat{f^{2}(i)}} .
$$

Following an idea from [CD-AD20, Section 3], we consider a Bernoulli action $\Gamma \curvearrowright D$ with abelian base and let $\mathcal{M}=D \rtimes \Gamma$. Let $\Psi: \mathcal{M} \rightarrow \mathcal{M} \bar{\otimes} M$ be the $*$-homomorphism given by $\Psi\left(d u_{g}\right)=d u_{g} \otimes u_{g}$, for all $d \in D$ and $g \in \Gamma$. From (4.4) we have that $M_{\hat{i}} \prec_{M}^{s} L\left(\Sigma_{i}\right)$, which gives $M_{\hat{i}} \prec_{\mathcal{M}}^{s, s^{\prime}} D \rtimes \Sigma_{i}$. By applying [Dr19, Lemma 2.3], we further derive that $\Psi\left(M_{\hat{i}}\right) \prec_{\mathcal{M} \bar{\otimes} M}^{s, s^{\prime}} \mathcal{M} \bar{\otimes} L\left(\Sigma_{i}\right)$. Next, by using [Dr19, Lemma 2.4] and the relation (4.5) we obtain that

$$
\Psi\left(M_{\widehat{i}}\right) \prec_{\mathcal{M} \bar{\otimes} M}^{s} \mathcal{M} \bar{\otimes} M_{\widehat{f^{2}(i)}} .
$$

Note that $i \in \widehat{f(i)}$. Using our assumption, we obtain that $\Delta\left(M_{i}\right) \prec_{M \bar{\otimes} M}^{s} M \bar{\otimes} M_{\widehat{f^{2}(i)}}$. Hence, by [DHI16, Remark 2.2] we get that

$$
\Psi\left(M_{i}\right) \prec_{\mathcal{M} \bar{\otimes} M}^{s} M \bar{\otimes} M_{\widehat{f^{2}(i)}} .
$$

Next, by using [Is19, Lemma 2.6], (4.6) and (4.7), we derive that $\Psi(M) \prec_{\mathcal{M} \bar{\otimes} M}^{s} \mathcal{M} \bar{\otimes} M_{\widehat{f^{2}(i)}}$. Since $\Psi(x)=x \otimes 1$, for any $x \in D$, we deduce that $\Psi(\mathcal{M}) \prec_{\mathcal{M} \bar{\otimes} M}^{s} \mathcal{M} \bar{\otimes} M_{\widehat{f^{2}(i)}}$. By [IPV10, Lemma 10.2] we obtain that $M \prec_{\mathcal{M}} M_{\widehat{f^{2}(i)}}$. Since $\mathcal{Q} \mathcal{N}_{\mathcal{M}}^{(1)}(M)=M$, we derive that $M \prec_{M} M_{\widehat{f^{2}(i)}}$, which implies that $M_{f^{2}(i)}$ is not diffuse, contradiction.

Hence, $\Delta\left(M_{\widehat{j}}\right) \prec_{M \bar{\otimes} M}^{s} M \bar{\otimes} M_{\widehat{j}}$, for any $j \in \overline{1, n}$. By applying [DHI16, Lemma 2.6(2)], the conclusion of the theorem follows. 


\section{Proofs of the MAIN RESUlts}

5.1. Proof of Theorem A, Before proceeding to the proof of Theorem $\mathrm{A}$, we need the following result.

Theorem 5.1. Let $M=M_{1} \bar{\otimes} \ldots \bar{\otimes} M_{n}$ be the tensor product of $n \geq 1 I I_{1}$ factors from $\mathcal{M} \cup \mathcal{M}_{w r}$. Assume $\Gamma$ is a countable icc group such that $M^{t}=L(\Gamma)$ for some $t>0$. Denote by $\Delta: M^{t} \rightarrow$ $M^{t} \bar{\otimes} M^{t}$ the $*$-homomorphism given by $\Delta\left(u_{g}\right)=u_{g} \otimes u_{g}$, for any $g \in \Gamma$.

Then for any $i \in \overline{1, n}$, there exists $f(i) \in \overline{1, n}$ such that $\Delta\left(M_{\hat{i}}^{t}\right) \prec_{M^{t} \bar{\otimes} M^{t}}^{s} M^{t} \bar{\otimes} M_{\widehat{f(i)}}^{t}$.

Proof. Let $\left(\tilde{M}_{i},\left(\alpha_{t}^{i}\right)_{t \in \mathbb{R}}\right)$ be an s-malleable deformation of $M_{i}$ given by the fact that $M_{i}$ belongs to $\mathcal{M} \cup \mathcal{M}_{w r}$, see Remark 3.8 (1). If $M_{i} \in \mathcal{M}$, we let $A_{i}$ be given as in the definition of Class $\mathcal{M}$. We naturally extend $\alpha_{t}^{i}$ to an automorphism $\alpha_{t}^{i} \in \operatorname{Aut}\left(M_{\bar{i}} \bar{\otimes} \tilde{M}_{i}\right)$. We may assume without loss of generality that $t=1$ since this simplification does not hide any essential part of the argument; note that for any $i \in \overline{1, n}$ there is a natural identification $M^{t}=M_{\bar{i}} \bar{\otimes} M_{i}^{t}$.

We first prove that there exists a function $f \in \overline{1, n} \rightarrow \overline{1, n}$ such that

$$
\Delta\left(M_{j}\right) \text { is non-amenable relative to } M \bar{\otimes} M_{\widehat{f(j)}} \text { for any } j \in \overline{1, n} \text {. }
$$

If (5.1) does not hold, then there exists $j \in \overline{1, n}$ such that $\Delta\left(M_{j}\right)$ is amenable relative to $M \bar{\otimes} M_{\hat{k}}$, for any $k \in \overline{1, n}$. By [PV11, Proposition 2.7], it follows that $\Delta\left(M_{j}\right)$ is amenable relative to $M \bar{\otimes} 1$. This further implies by [PV10, Proposition 7.2(4)] that $M_{j}$ is amenable, contradiction. Therefore, there exists a function $f$ that satisfies (5.1). Next, we show that

$$
\Delta\left(M_{\widehat{j}}\right) \prec_{M \bar{\otimes} M} M \bar{\otimes} M_{\widehat{f(j)}} \text {, for any } j \in \overline{1, n} \text {. }
$$

To show this, fix $j \in \overline{1, n}$ and notice first that $\mathcal{N}_{M \bar{\otimes} M}\left(\Delta\left(M_{j}\right)\right)^{\prime} \cap M \bar{\otimes} M=\mathbb{C} 1$ since $\Gamma$ is icc. Thus, using [DHI16, Lemma 2.6(2)], we obtain from (5.1) that

$$
\Delta\left(M_{j}\right) \text { is strongly non-amenable relative to } M \bar{\otimes} M_{\widehat{f(j)}} \text {. }
$$

Case 1. $M_{f(j)} \in \mathcal{M}$.

In this case it follows directly from (5.3) that id $\otimes \alpha_{t}^{f(j)} \rightarrow$ id uniformly on $\left(\Delta\left(M_{\widehat{j}}\right)\right)_{1}$. Assume by contradiction that $\Delta\left(M_{\widehat{j}}\right) \nprec_{M \bar{\otimes} M} M \bar{\otimes} M_{\widehat{f(j)}}$. If $\Delta\left(M_{\widehat{j}}\right) \nprec_{M \bar{\otimes} M} M \bar{\otimes}\left(M_{\widehat{f(j)}} \bar{\otimes} A_{f(j)}\right)$, we obtain from Theorem 3.2(1) that id $\otimes \alpha_{t}^{f(j)} \rightarrow$ id uniformly on $(\Delta(M))_{1}$. This shows that $\alpha_{t}^{f(j)} \rightarrow$ id uniformly on $(M)_{1}$, and hence on $M_{f(j)}$, contradiction. Therefore, $\Delta\left(M_{\widehat{j}}\right) \prec_{M \bar{\otimes} M} M \bar{\otimes}\left(M_{\widehat{f(j)}} \bar{\otimes} A_{f(j)}\right)$. Using condition (2.ii) from Class $\mathcal{M}$, it follows that (5.2) holds in this case.

Case 2. $M_{f(j)} \in \mathcal{M}_{w r}$.

In this case we can write $M_{f(j)}=B_{f(j)} \rtimes \Lambda_{f(j)}$ as the von Neumann algebra of a generalized Bernoulli action as in Class $\mathcal{M}_{w r}$. By (5.3) and [BV12, Theorem 3.1] we have that id $\otimes \alpha_{t}^{f(j)} \rightarrow$ id uniformly on $\left(\Delta\left(M_{\widehat{j}}\right)\right)_{1}$. Using [IPV10, Theorem 4.2] we obtain that $\Delta\left(M_{\widehat{j}}\right) \prec_{M \bar{\otimes} M} M \bar{\otimes} M_{\widehat{f(j)}}$ or $\Delta(M) \prec_{M \bar{\otimes} M} M \bar{\otimes}\left(M_{\widehat{f(j)}} \bar{\otimes} L\left(\Lambda_{f(j)}\right)\right)$ or $\Delta(M) \prec_{M \bar{\otimes} M} M \bar{\otimes}\left(M_{\widehat{f(j)}} \bar{\otimes}\left(B_{f(j)} \rtimes \theta_{f(j)}\right)\right)$, where $\theta_{f(j)}<$ $\Lambda_{f(j)}$ is an infinite index subgroup. From [IPV10, Proposition 7.2] the last two possibilities give a contradiction. This shows that (5.2) holds.

Finally, by using [DHI16, Lemma 2.4(2)], we end the proof of the theorem.

We are now ready to prove the following result which is a generalization of Theorem $\mathrm{A}$, 
Theorem 5.2. Let $M=M_{1} \bar{\otimes} \ldots \bar{\otimes} M_{n}$ be the tensor product of $n \geq 1 I I_{1}$ factors from $\mathcal{M} \cup \mathcal{M}_{w r}$. Assume $\Gamma$ is a countable icc group such that $M^{t}=L(\Gamma)$ for some $t>0$.

Then there exist a product decomposition $\Gamma=\Gamma_{1} \times \cdots \times \Gamma_{n}$, a unitary $u \in M$ and some positive numbers $t_{1}, \ldots, t_{n}$ with $t_{1} \cdots t_{n}=t$ such that $M_{i}^{t_{i}}=u L\left(\Gamma_{i}\right) u^{*}$, for any $i \in \overline{1, n}$.

Proof. Let $\left(\tilde{M}_{i},\left(\alpha_{t}^{i}\right)_{t \in \mathbb{R}}\right)$ be an s-malleable deformation of $M_{i}$ given by the fact that $M_{i}$ belongs to Class $\mathcal{M} \cup \mathcal{M}_{w r}$, see Remark $3.8(1)$. If $M_{i} \in \mathcal{M}$, we let $A_{i}$ be given as in the definition of Class $\mathcal{M}$. We naturally extend $\alpha_{t}^{i}$ to an automorphism $\alpha_{t}^{i} \in \operatorname{Aut}\left(M_{\hat{i}} \bar{\otimes} \tilde{M}_{i}\right)$. Next, we may assume that $t=1$ since this simplification does not hide any essential part of the argument.

Following [PV09], we denote by $\Delta: M \rightarrow M \bar{\otimes} M$ the $*$-homomorphism given by $\Delta\left(u_{g}\right)=u_{g} \otimes u_{g}$, for any $g \in \Gamma$. By Theorem 5.1 and Theorem 4.3 , we have that

$$
\Delta\left(M_{I}\right) \prec_{M \bar{\otimes} M}^{s} M \bar{\otimes} M_{I} \text {, for any subset } I \subset \overline{1, n} .
$$

Our goal is to prove the following claim.

Claim. There exist a subgroup $\Sigma<\Gamma$ and a non-zero projection $f \in L(\Sigma)^{\prime} \cap M$ such that $M_{\widehat{n}} \prec_{M} L(\Sigma) f$ and $L(\Sigma) f \prec_{M}^{s} M_{\widehat{n}}$.

Proof of Claim. Using Theorem 4.2, we can find a subgroup $\Sigma<\Gamma$ with non-amenable centralizer $C_{\Gamma}(\Sigma)$ such that $M_{\hat{n}} \prec_{M} L(\Sigma)$ and $L(\Sigma) \prec_{M} M_{\hat{n}}$. By using [DHI16, Lemma 2.4(4)] there exists a non-zero projection $f \in L\left(\Sigma C_{\Gamma}(\Sigma)\right)^{\prime} \cap M$ such that

$$
M_{\hat{n}} \prec_{M}^{s^{\prime}} L(\Sigma) f \text { and } L(\Sigma) \prec_{M} M_{\hat{n}} \text {. }
$$

Next, by using [Va08, Lemma 3.5] and [DHI16, Lemma 2.6(3)] we deduce from (5.5) that $L\left(C_{\Gamma}(\Sigma)\right) f$ is amenable relative to $M_{n}$. Since $C_{\Gamma}(\Sigma)$ is non-amenable, we derive from [PV11, Proposition 2.7] that $L\left(C_{\Gamma}(\Sigma)\right) f$ is non-amenable relative to $M_{\widehat{n}}$. Using [DHI16, Lemma 2.4] there exists a non-zero projection $f_{1} \in L\left(\Sigma C_{\Gamma}(\Sigma)\right)^{\prime} \cap M$ with $f_{1} \leq f$ such that

$$
L\left(C_{\Gamma}(\Sigma)\right) f_{1} \text { is strongly non-amenable relative to } M_{\widehat{n}} \text {. }
$$

Next, we note that

$$
\alpha_{t}^{n} \rightarrow \text { id uniformly on }\left(L(\Sigma) f_{1}\right)_{1} \text {. }
$$

Indeed, if $M_{n}$ belongs to $\mathcal{M}$, this follows immediately, while if $M$ belongs to $\mathcal{M}_{w r}$, this follows from [BV12, Theorem 3.1]. Next, remark that the claim would follow if $L(\Sigma) f_{1} \prec_{M} M_{\widehat{n}}$ because we could use [DHI16, Lemma 2.4] and derive that there exists a non-zero projection $f_{0} \in L(\Sigma)^{\prime} \cap M$ with $f_{0} \leq f_{1}$ such that $L(\Sigma) f_{0} \prec_{M}^{s} M_{\hat{n}}$.

Hence, we assume by contradiction that $L(\Sigma) f_{1} \nprec_{M} M_{\widehat{n}}$.

Following CdSS15, Section 4], we let $\Omega=\left\{g \in \Gamma \mid \mathcal{O}_{\Sigma}(g)\right.$ is finite $\}$, where $\mathcal{O}_{\Sigma}(g)=\left\{h g h^{-1} \mid h \in \Sigma\right\}$, and notice that $L(\Sigma)^{\prime} \cap M \subset L(\Omega)$. We continue by proving the following.

Subclaim. There exists a non-zero projection $f_{2} \in L(\Omega)^{\prime} \cap M$ such that

$$
\alpha_{t}^{n} \rightarrow \text { id uniformly on }\left(L(\Omega) f_{2}\right)_{1} \text {. }
$$

Proof of subclaim. We split the proof of the subclaim in two parts.

Case 1. $M_{n}$ belongs to Class $\mathcal{M}$.

Note that there exists a sequence of increasing subgroups $\Omega_{i}<\Omega$ such that $\Omega=\vee_{i \geq 1} \Omega_{i}$ and by letting $\Sigma_{i}=C_{\Sigma}\left(\Omega_{i}\right)$, we have $\left[\Sigma: \Sigma_{i}\right]<\infty$, for any $i \geq 1$. Indeed, let $\left\{\mathcal{O}_{i}\right\}_{i \geq 1}$ be a countable enumeration of all the finite orbits of the action by conjugation of $\Sigma$ on $\Gamma$. Notice that $\Omega_{i}:=$ $\left\langle\cup_{j=1}^{i} \mathcal{O}_{j}\right\rangle<\Omega, i \geq 1$, is an ascending sequence of subgroups with $\Omega=\vee_{i \geq 1} \Omega_{i}$. Since $\cup_{j=1}^{i} \mathcal{O}_{j} \subset \Omega$ is 
a finite set, it follows that $\Sigma_{i}:=\cap_{g \in \cup_{j=1}^{i}} \mathcal{O}_{j} C_{\Sigma}(g)=C_{\Sigma}\left(\Omega_{i}\right)$ is a decreasing sequence of finite index subgroups of $\Sigma$.

Next, note that $f_{1} \in L\left(\Sigma_{i}\right)^{\prime} \cap M$ and $L\left(\Sigma_{i}\right) f_{1} \nprec_{M} M_{\hat{n}}$ since $\left[\Sigma: \Sigma_{i}\right]<\infty$, for any $i \geq 1$. Moreover, we note that $L\left(\Sigma_{i}\right) f_{1} \nprec_{M} M_{\hat{n}} \bar{\otimes} A_{n}$. Otherwise, by using condition (2) in Class $\mathcal{M}$, we get that there exists a non-zero projection $f_{2} \in\left(L\left(\Sigma_{i}\right)^{\prime} \cap M\right)^{\prime} \cap M \subset L\left(C_{\Gamma}(\Sigma)\right)^{\prime} \cap M$ with $f_{2} \leq f_{1}$ such that $\left(L\left(\Sigma_{i}\right)^{\prime} \cap M\right) f_{2}$ is amenable relative to $M_{\widehat{n}}$, which contradicts (5.6).

Therefore, since $M \subset M_{\widehat{n}} \bar{\otimes} \tilde{M}_{n}$ is mixing relative to $M_{\widehat{n}} \bar{\otimes} A_{n}$ we obtain from (5.7) and Theorem $3.2(1)$ that

$$
\alpha_{t}^{n} \rightarrow \text { id uniformly on }\left(f_{1}\left(L\left(\Sigma_{i}\right) \vee\left(L\left(\Sigma_{i}\right)^{\prime} \cap M\right)\right) f_{1}\right)_{1} \text {, for any } i \geq 1 .
$$

Next, since $A_{n}$ is amenable, we notice that (5.6) together with [DHI16, Theorem 2.6(3)] imply that $\left.f_{1}\left(L\left(\Sigma_{i}\right)^{\prime} \cap M\right)\right) f_{1} \nprec_{M} M_{\widehat{n}} \bar{\otimes} A_{n}$ for any $i \geq 1$. We can therefore combine (5.8) with Theorem $3.2(2)$ and deduce that

$$
\alpha_{t}^{n} \rightarrow \text { id uniformly on }\left(f_{1}\left(\bigvee_{i \geq 1}\left(L\left(\Sigma_{i}\right)^{\prime} \cap M\right)\right) f_{1}\right)_{1} .
$$

We can apply Proposition 3.3 and derive that if we denote by $f_{2}$ the central support of $f_{1}$ in $\bigvee_{i \geq 1}\left(L\left(\Sigma_{i}\right)^{\prime} \cap M\right)$, we further obtain from that

$$
\alpha_{t}^{n} \rightarrow \text { id uniformly on }\left(\left(\bigvee_{i \geq 1}\left(L\left(\Sigma_{i}\right)^{\prime} \cap M\right)\right) f_{2}\right)_{1}
$$

Since $L(\Omega) \subset \bigvee_{i \geq 1} L\left(\Sigma_{i}\right)^{\prime} \cap M$ and $f_{2} \in \mathcal{Z}\left(\bigvee_{i \geq 1}\left(L\left(\Sigma_{i}\right)^{\prime} \cap M\right)\right)$, it follows that the subclaim is proven in this case.

Case 2. $M_{n}$ belongs to Class $\mathcal{M}_{w r}$.

We can write $M_{n}=B_{n} \rtimes \Lambda_{n}$ where $\Lambda_{n} \curvearrowright B_{n}$ is a generalized Bernoulli action with amenable base as given by Class $\mathcal{M}_{w r}$. For proving the subclaim, we follow a slightly different approach than the one used in Case 1. Using Popa's compression formulas for quasi-normalizers [Po03], we have

$$
\mathcal{Q N}_{f_{1} M f_{1}}\left(L(\Sigma) f_{1}\right)=f_{1} \mathcal{Q N}_{M}(L(\Sigma)) f_{1} .
$$

By applying [IPV10, Theorem 4.2] to (5.7), we derive that (i) $L(\Sigma) f_{1} \prec_{M} M_{\widehat{n}}$ or (ii) $L\left(Q N_{\Gamma}(\Sigma)\right) f_{1}$ $\prec_{M} M_{\widehat{n}} \bar{\otimes}\left(B_{n} \rtimes \theta_{n}\right)$, where $\theta_{n}<\Lambda_{n}$ is an infinite index subgroup, or (iii) there exists a partial isometry $w \in M$ with $w w^{*}=f_{1}$ and $w^{*} \mathcal{Q N}_{f_{1} M f_{1}}\left(L(\Sigma) f_{1}\right)^{\prime \prime} w \subset M_{\widehat{n}} \bar{\otimes} L\left(\Lambda_{n}\right)$. Option (i) is not possible since we assumed by contradiction that $L(\Sigma) f_{1} \nprec_{M} M_{\widehat{n}}$. We now show that option (ii) leads to a contradiction as well. Note that by passing to relative commutants in (5.5), we derive that $M_{n} \prec_{M} L(\Omega)$. Since $\Omega \subset Q N_{\Gamma}(\Sigma)$, option (ii) implies that $L(\Omega) \prec_{M} M_{\widehat{n}} \bar{\otimes}\left(B_{n} \rtimes \theta_{n}\right)$. Combining all these with (5.5), we can apply Proposition 2.6 and derive that $\left[\Lambda_{n}: \theta_{n}\right]<\infty$, contradiction.

Next, note that option (iii) combined with (5.9) implies that

$$
\alpha_{t}^{n} \rightarrow \text { id uniformly on }\left(f_{1} \mathcal{Q N}_{M}(L(\Sigma))^{\prime \prime} f_{1}\right)_{1} \text {. }
$$

Since $\Omega \subset Q N_{\Gamma}(\Sigma)$, the subclaim follows by using Proposition 3.3.

Following the augmentation technique from [CD-AD20, Section 3], we consider a Bernoulli action $\Gamma \curvearrowright D$ with abelian base. Denote $\mathcal{M}=D \rtimes \Gamma$ and let $\Psi: \mathcal{M} \rightarrow \mathcal{M} \bar{\otimes} M$ be the $*$-homomorphism given by $\Psi\left(d u_{g}\right)=d u_{g} \otimes u_{g}$, for all $d \in D$ and $g \in \Gamma$. Next, by passing to relative commutants in (5.5) we obtain that $M_{n} \prec_{M} L(\Omega)$, which implies that $M_{n} \prec_{\mathcal{M}}^{s^{\prime}} D \rtimes \Omega$. This shows using Dr19, Lemma 2.3] that $\Psi\left(M_{n}\right) \prec_{\mathcal{M} \bar{\otimes} M}^{s^{\prime}} \mathcal{M} \bar{\otimes} L(\Omega)$. In particular, $\Psi\left(M_{n}\right) \prec_{\mathcal{M} \bar{\otimes} M} \mathcal{M} \bar{\otimes} L(\Omega) f_{2}$.

Hence, there exist some projections $p \in M_{n}, q \in \mathcal{M} \bar{\otimes} L(\Omega) f_{2}$, a non-zero partial isometry $w \in$ $q(\mathcal{M} \bar{\otimes} M) \Psi(p)$ and a $*$-homomorphism $\theta: \Psi\left(p M_{n} p\right) \rightarrow q\left(\mathcal{M} \bar{\otimes} L(\Omega) f_{2}\right) q$ such that $\theta(x) w=w x$, for any $x \in \Psi\left(p M_{n} p\right)$. Let $\tilde{p}=\Psi(p)$ and note that $w^{*} w \in \Psi\left(p M_{n} p\right)^{\prime} \cap \tilde{p}(\mathcal{M} \bar{\otimes} M) \tilde{p}$. Therefore, by using 
the multiplicativity of id $\otimes \alpha_{t}^{n}$ and its pointwise $\|\cdot\|_{2}$-convergence to the identity, the subclaim implies that

$$
\text { id } \otimes \alpha_{t}^{n} \rightarrow \text { id uniformly on }\left(\Psi\left(p M_{n} p\right) w^{*} w\right)_{1} \text {. }
$$

The claim will be proven by considering again two separate cases.

Case 1. $M_{n}$ belongs to Class $\mathcal{M}$.

If $\Psi\left(p M_{n} p\right) w^{*} w \nprec_{\mathcal{M}} \bar{\otimes} M \mathcal{M} \bar{\otimes}\left(M_{\hat{n}} \bar{\otimes} A_{n}\right)$, then Theorem $3.2(1)$ and (5.10) give

$$
\text { id } \otimes \alpha_{t}^{n} \rightarrow \text { id uniformly on }\left(w^{*} w\left(\Psi\left(p M_{n} p\right) \vee \Psi\left(p M_{n} p\right)^{\prime} \cap \tilde{p}(\mathcal{M} \bar{\otimes} M) \tilde{p}\right) w^{*} w\right)_{1} .
$$

Let $\tilde{p}_{1}$ be the central support of $w^{*} w$ in $\Psi\left(M_{n}\right) \vee \Psi\left(M_{n}\right)^{\prime} \cap(\mathcal{M} \bar{\otimes} M)$ and note that $\tilde{p}_{1} \in \Psi(M)^{\prime} \cap$ $(\mathcal{M} \bar{\otimes} M)=\mathbb{C} 1$ since $\Gamma$ is icc. By using Proposition (3.3), we further obtain that id $\otimes \alpha_{t}^{n} \rightarrow$ id uniformly on $(\Psi(M))_{1}$, which shows that $\alpha_{t}^{n} \rightarrow$ id uniformly on $(M)_{1}$, contradiction.

Hence, $\Psi\left(p M_{n} p\right) w^{*} w \prec_{\mathcal{M} \bar{\otimes} M} \mathcal{M} \bar{\otimes}\left(M_{\hat{n}} \bar{\otimes} A_{n}\right)$, and therefore $\Psi\left(M_{n}\right) \prec_{\mathcal{M} \bar{\otimes} M}^{s} \mathcal{M} \bar{\otimes}\left(M_{\hat{n}} \bar{\otimes} A_{n}\right)$ by [DHI16, Lemma 2.4(2)]. Relation (5.4) implies in particular that $\Psi\left(M_{n}\right) \prec_{\mathcal{M} \bar{\otimes} M}^{s} \mathcal{M} \bar{\otimes} M_{n}$. By using [DHI16, Lemma 2.8(2)], we derive that $\Psi\left(M_{n}\right) \prec_{\mathcal{M} \bar{\otimes} M}^{s} \mathcal{M} \bar{\otimes} A_{n}$, which implies by [PV10, Lemma 10.2] that $M_{n}$ is amenable, contradiction. Hence, the claim is proven in this case.

Case 2. $M_{n}$ belongs to Class $\mathcal{M}_{w r}$.

As before, we assume $M_{n}=B_{n} \rtimes \Lambda_{n}$ where $\Lambda_{n} \curvearrowright C_{n}^{I_{n}}=: B_{n}$ is a generalized Bernoulli action with amenable base as given by Class $\mathcal{M}_{w r}$. If $\Psi\left(p M_{n} p\right) w^{*} w \nprec_{\mathcal{M} \bar{\otimes} M} \mathcal{M} \bar{\otimes} M_{\hat{n}}$, we derive from [IPV10, Theorem 4.2] and (5.10) that $\Psi(M) \prec_{\mathcal{M} \bar{\otimes} M} \mathcal{M} \bar{\otimes}\left(B_{n} \rtimes \theta_{n}\right)$, where $\theta_{n}<\Lambda_{n}$ is an infinite index subgroup, or $\Psi(M) \prec_{\mathcal{M} \bar{\otimes} M} \mathcal{M} \bar{\otimes} L\left(\Lambda_{n}\right)$, and therefore, $\Psi(\mathcal{M}) \prec_{\mathcal{M} \bar{\otimes} M} \mathcal{M} \bar{\otimes}\left(B_{n} \rtimes \theta_{n}\right)$ or $\Psi(\mathcal{M}) \prec_{\mathcal{M} \bar{\otimes} M} \mathcal{M} \bar{\otimes} L\left(\Lambda_{n}\right)$, respectively. By using [IPV10, Lemma 10.2] it is easy to see that we would get a contradiction. Hence, $\Psi\left(p M_{n} p\right) w^{*} w \prec_{\mathcal{M} \bar{\otimes} M} \mathcal{M} \bar{\otimes} M_{\hat{n}}$, and by proceeding as in the last paragraph of Case 1 above, we obtain that $\Psi\left(M_{n}\right) \prec_{\mathcal{M} \otimes M}^{s} \mathcal{M} \otimes 1$, which is again a contradiction.

Finally, note that (5.4) implies in particular that $\Delta\left(M_{1}\right) \prec_{M \bar{\otimes} M} M \bar{\otimes} M_{1}$. Using Theorem 4.2 , we obtain a subgroup $\Theta<\Gamma$ such that $M_{1} \prec_{M} L(\Theta)$ and $L(\Theta) \prec_{M} M_{1}$. In combination with the claim, it follows from Theorem 2.3 that there exist a product decomposition $\Gamma=\Gamma_{1}^{n-1} \times \Gamma_{n}$, a decomposition $M=M_{\widehat{n}}^{s} \bar{\otimes} M_{n}^{1 / s}$ for some $s>0$ and a unitary $u \in \mathcal{U}(M)$ such that $u M_{\widehat{n}}^{s} u^{*}=L\left(\Gamma_{1}^{n-1}\right)$ and $u M_{n}^{1 / s} u^{*}=L\left(\Gamma_{n}\right)$. Therefore, we obtain the conclusion of the theorem by a simple induction argument.

5.2. Proof of Corollary B. Let $\theta: L(\Gamma)^{t} \rightarrow L(\Lambda)$ be a $*$-ismorphism where $\Lambda$ is any countable group and $t>0$. By Theorem $\mathrm{A}$, there exist a product decomposition $\Lambda=\Lambda_{1} \times \cdots \times \Lambda_{n}$, some positive numbers $t_{1}, \ldots, t_{n}>0$ with $t_{1} \cdots t_{n}=t$ and a unitary $w \in L(\Lambda)$ such that $\theta\left(L\left(\Gamma_{i}\right)^{t_{i}}\right)=$ $w L\left(\Lambda_{i}\right) w^{*}$, for any $i \in \overline{1, n}$. Since $\Gamma_{i}$ is $\mathrm{W}^{*}$-superrigid, it follows that $t_{i}=1$ and there exist a group isomorphism $\delta_{i}: \Gamma_{i} \rightarrow \Lambda_{i}$, a unitary $w_{i} \in L\left(\Lambda_{i}\right)$ and a character $\omega_{i}: \Gamma_{i} \rightarrow \mathbb{T}$ such that $\theta\left(u_{g}\right)=\omega_{i}(g) w_{i} v_{\delta(g)} w_{i}^{*}$, for all $i \in \overline{1, n}$ and $g \in \Gamma_{i}$. Hence, $t=1$ and by letting $\omega=\prod_{i=1}^{n} \omega_{i}$, $\delta=\prod_{i=1}^{n} \delta_{i}$ and $u=\prod_{i=1}^{n} w_{i}$, we get the desired conclusion. Finally, we notice that any group from $\mathcal{I P \mathcal { V }}$ is $\mathrm{W}^{*}$-superrigid by [PV10, Theorem 8.3].

5.3. Proof of Corollary C. On one hand, note that if $\Gamma_{0}$ is an icc non-amenable countable group with $\beta_{1}^{(2)}\left(\Gamma_{0}\right)>0$, then its amenable radical is trivial, i.e. any normal amenable subgroup of $\Gamma_{0}$ is trivial. On the other hand, note that any group from $\mathcal{A}_{2}$ has trivial amenable radical by using [CD-AD20, Proposition 6.3]. 
Therefore, $\Gamma$ has trivial amenable radical, so by using [BKKO14, Theorem 1.3], it follows that $C_{r}^{*}(\Gamma)$ has a unique trace. This implies that any $*$-isomorphism $\theta: C_{r}^{*}(\Gamma) \rightarrow C_{r}^{*}(\Lambda)$ extends to a *-isomorphism $\theta: L(\Gamma) \rightarrow L(\Lambda)$. The conclusion now follows from Theorem $\mathrm{A}$.

\section{REFERENCES}

[Be14] M. Berbec: $W^{*}$-superrigidity for wreath products with groups having positive first $\ell^{2}$-Betti number, Internat. J. Math 26 (2015), no. 1, 1550003, 27pp.

[BKKO14] E. Breuillard, M. Kalantar, M. Kennedy, N. Ozawa, ${ }^{*}$-Simplicity. Publ. d' IHES.

[Bo14] R. Boutonnet: Plusieurs aspects de rigidite des algebres de von Neumann, PhD thesis (2014).

[BMO19] J. Bannon, A. Marrakchi N.Ozawa: Full factors and co-amenable inclusions, Comm. Math. Phys., 378 (2020), 1107-1121.

[BV12] M. Berbec, S. Vaes: $W^{*}$-superrigidity for group von Neumann algebras of left-right wreath products, Proceedings of the London Mathematical Society (3) 108 (2014), no. 5, 1116-1152.

[CD-AD20] I.Chifan, A. Diaz-Arias, D. Drimbe: New examples of $W^{*}$ and $C^{*}$-superrigid groups, preprint arXiv:2010.01223.

[CD-AD21] I. Chifan, A. Diaz-Arias, D. Drimbe: $W^{*}$ and $C^{*}$-superrigidity results for coinduced groups, preprint arXiv:2107.05976.

[CDHK20] I. Chifan, S. Das, C. Houdayer, K. Khan: Examples of property (T) II factors with trivial fundamental group, preprint arXiv:2003.08857.

[CDK19] I. Chifan, S. Das and K. Khan, Some Applications of Group Theoretic Rips Constructions to the Classification of von Neumann Algebras, https://arxiv.org/pdf/1911.11729.

[CdSS15] I. Chifan, R. de Santiago, T. Sinclair: $W^{*}$-rigidity for the von Neumann algebras of products of hyperbolic groups, Geom. Funct. Anal. 26 (2016), no. 1, 136-159.

[CI08] I. Chifan, A. Ioana: Ergodic subequivalence relations induced by a Bernoulli action, Geom. Funct. Anal. 20 (2010), no. 1, 53-67.

[CI17] I. Chifan, A. Ioana: Amalgamated Free Product Rigidity for Group von Neumann Algebras. Adv. Math. 329 (2018).

[Co76] A. Connes: Classification of injective factors, Ann. Math. 104 (1976) 73-115.

[CU18] I. Chifan, B. Udrea: Some rigidity results for II $I_{1}$ factors arising from wreath products of property (T) groups, preprint arXiv:1804.04558.

[DHI16] D. Drimbe, D. Hoff, A. Ioana: Prime II factors arising from irreducible lattices in product of rank one simple Lie groups, to appear in J. Reine. Angew. Math, arXiv: 1611.02209 (2016).

[Dr17] D. Drimbe: $W^{*}$-superrigidity for coinduced actions Internat. J. Math. 29 (2018), no. 4, 1850033,20 pp.

[Dr19] D. Drimbe: Orbit equivalence rigidity for product actions, Comm. Math. Phys. 379 (2020), 41-59.

[Dr20] D. Drimbe: Solid ergodicity and orbit equivalence rigidity for coinduced actions, to appear in Int. Math. Res. Not.

[dSHHS20] R. de Santiago, B. Hayes, D. Hoff, T. Sinclair: Maximal rigid subalgebras of deformations and $L^{2}-$ cohomology, to appear in Analysis and PDE.

[ER18] C. Eckhardt, S. Raum: $C^{*}$-superrigidity of 2-step nilpotent groups, Adv. Math. 338 (2018), 175-195, DOI 10.1016/j.aim.2018.09.008.

[Ho15] D. Hoff: Von Neumann algebras of equivalence relations with nontrivial one-cohomology, J. Funct. Anal. 270 (2016), no. 4, 1501-1536.

[HU15] C. Houdayer, Y. Ueda: Rigidity of free product von Neumann algebras, Compos. Math. 152 (2016) 24612492 .

[Io06] A. Ioana: Rigidity results for wreath product of $I I_{1}$ factors, J. Funct. Anal. 252 (2007), 763-791.

[Io11] A. Ioana: Uniqueness of the group measure space decomposition for Popa's $\mathcal{H} \mathcal{T}$ factors, Geom. Funct. Anal. 22 (2012), no. 3, 699-732.

[Io12a] A. Ioana: Cartan subalgebras of amalgamated free product $I I_{1}$ factors, With an appendix by Adrian Ioana and Stefaan Vaes, Ann. Sci. Ec. Norm. Sup. (4) 48 (2015), no. 1, 71-130.

[Io12b] A. Ioana: Classification and rigidity for von Neumann algebras, European Congress of Mathematics, EMS (2013), 601-625.

[Io17] A. Ioana: Rigidity for von Neumann algebras, Proc. Int. Cong. of Math. 2018 Rio de Janeiro, Vol. 2 (16351668).

[IM19] Y. Isono, A. Marrakchi: Tensor product decompositions and rigidity of full factors, To appear in Ann. Sci. Ec. Norm. Super. 
[IPP05] A. Ioana, J. Peterson, S. Popa: Amalgamated free products of weakly rigid factors and calculation of their symmetry groups, Acta Math. 200 (2008), 85-153.

[IPV10] A. Ioana, S. Popa, S. Vaes: A class of superrigid group von Neumann algebras, Ann. of Math. (2) 178 (2013), no. 1, 231-286.

[Is19] Y. Isono: On fundamental groups of tensor product II 1 factors. J. Inst. Math. Jussieu 19 (2020), no. 4, 1121-1139.

[KV15] A. Krogager, S. Vaes: A class of II 1 factors with exactly two crossed product decompositions, preprint arXiv:1512.06677, to appear in J. Mathématiques Pures et Appliquées.

[MvN43] F.J. Murray, J. von Neumann: Rings of operators, IV. Ann. Math. 44 (1943), 716-808

[Oz03] N. Ozawa: Solid von Neumann algebras, Acta Math. 192 (2004), no. 1, 111-117.

[OP03] N. Ozawa, S. Popa: Some prime factorization results for type $I I_{1}$ factors, Invent. Math. 156 (2004), no. 2, 223-234. pp.

[OP07] N. Ozawa, S. Popa: On a class of $I_{1}$ factors with at most one Cartan subalgebra, Ann. of Math. (2) 172 (2010), no. 1, 713-749.

[Po99] S. Popa, Some properties of the symmetric enveloping algebra of a factor, with applications to amenability and property (T), Doc. Math. 4 (1999), 665-744.

[Po01] S. Popa: On a class of type II factors with Betti numbers invariants, Ann. of Math. 163 (2006), 809-899.

[Po03] S. Popa: Strong rigidity of $I I_{1}$ factors arising from malleable actions of w-rigid groups. I., Invent. Math. 165 (2006), no. 2, 369-408.

[Po04] S. Popa: Strong rigidity of $I_{1} 1$ factors arising from malleable actions of w-rigid groups, II. Invent. Math. 165 (2006), 409-452.

[Po05] S. Popa: Cocycle and orbit equivalence superrigidity for malleable actions of w-rigid groups, Invent. Math. 170 (2007), 243-295.

[Po06a] S. Popa: On the superrigidity of malleable actions with spectral gap, J. Amer. Math. Soc. 21 (2008), 9811000 .

[Po06b] S. Popa: On Ozawa's property for free group factors, Int. Math. Res. Not. 2007, no. 11, Art. ID rnm036, 10 pp.

[Po07] S. Popa: Deformation and rigidity for group actions and von Neumann algebras, In Proceedings of the ICM (Madrid, 2006), Vol. I, European Mathematical Society Publishining House, 2007, 445-477.

[PS09] J. Peterson, T. Sinclair: On cocycle superrigidity for Gaussian actions Erg. Th. and Dyn. Sys.32 (2012), no. 1, 249-272.

[PV09] S. Popa, S. Vaes: Group measure space decomposition of $I I_{1}$ factors and $W^{*}$-superrigidity, Invent. Math. 182 (2010), no. 2, 371-417.

[PV11] S. Popa, S. Vaes: Unique Cartan decomposition for $I I_{1}$ factors arising from arbitrary actions of free groups, Acta Math. 212 (2014), no. 1, 141-198.

[Sc74] S. Scheinberg: Homeomorphism and isomorphism of abelian groups, Can. J. Math., 26(6):1515-1519, 1974.

[Si10] T. Sinclair: Strong solidity of group factors from lattices in $S O(n, 1)$ and $S U(n, 1)$, J. Funct. Anal., Volume 260 (2011), no.11, 3209-3221.

[SW11] O. Sizemore, A. Winchester: Unique prime decomposition results for factors coming from wreath product groups, Pacific J. Math. 265 (2013), 221-232.

[Va08] S. Vaes: Explicit computations of all finite index bimodules for a family of II $I_{1}$ factors, Ann. Sci. Éc. Norm. Supér. (4) 41 (2008), no. 5, 743-788.

[Va10a] S. Vaes: Rigidity for von Neumann algebras and their invariants, Proceedings of the ICM (Hyderabad, India, 2010), Vol. III, Hindustan Book Agency (2010), 1624-1650.

[Va10b] S. Vaes: One-cohomology and the uniqueness of the group measure space decomposition of a II $I_{1}$ factor, Math. Ann. 355 (2013), no. 2, 661-696.

Department of Mathematics, Ku Leuven, Celestijnenlaan 200B, B-3001 Leuven, Belgium

Email address: daniel.drimbe@kuleuven.be 